\title{
Tight Fluctuations of Weight-Distances in Random Graphs with Infinite-Variance Degrees
}

\author{
Enrico Baroni ${ }^{1} \cdot$ Remco van der Hofstad ${ }^{1} \cdot$ Júlia Komjáthy $^{1}$ (ib
}

Received: 23 September 2016 / Accepted: 12 December 2018 / Published online: 7 January 2019

(c) The Author(s) 2019

\begin{abstract}
We prove results for first-passage percolation on the configuration model with degrees having asymptotic finite mean, infinite variance and i.i.d. edge-weights with strictly positive support of the form $Y=a+X$, where $a$ is a positive constant and the excess edge-weight $X$ is a non-negative random variable with zero as the infimum of its support. We prove that the weight of the optimal path between two uniformly chosen vertices has tight fluctuations around the asymptotic mean of the graph-distance if and only if the following condition holds: the random variable $X$ is such that the age-dependent branching process describing first-passage percolation exploration in the same graph with edge-weights from distribution $X$ has a positive probability to reach infinitely many individuals in a finite time. This shows that almost-shortest paths in the graph-distance proliferate, in the sense that there even exist paths having tight total excess edge-weight for appropriate edge-weight distributions. Our proof makes use of a degree-dependent percolation model that we believe is interesting in its own right, as well as tightness results for distances in scale-free configuration models that we prove to hold under rather weak conditions on the degrees.
\end{abstract}

Keywords Configuration model · Power law degrees · Weighted typical distances · First passage percolation

Mathematics Subject Classification Primary 05C80 - 05C82 - 90B15 - 60C05 - 60D05

Júlia Komjáthy

j.komjathy@ tue.nl

Enrico Baroni

e.baroni@tue.nl

Remco van der Hofstad

r.w.v.d.hofstad@TUE.nl

1 Department of Mathematics and Computer Science, Eindhoven University of Technology,

Eindhoven, Netherlands 


\section{Introduction}

\subsection{Motivations}

First Passage Percolation (FPP) has been introduced as a model for the spread of a material in a random medium (see [10]). In more recent times, motivated by the boost in interest in complex networks and the related random graph models for them, it has appeared as a mathematical tool for studying dynamics in complex networks. A typical setting in this sense is a transportation network in which roads, corresponding to edges, have certain transport times, corresponding to weights on the edges (see [13]). Typical travel times are then affected by the number of edges in the almost-shortest paths between the vertices of the network and the passage time cost to cross the edges. The corresponding mathematical model is a simple and connected graph $G$, such that every edge $e$ has a random variable $Y_{e}$ assigned to it, representing the passage time through the edge $e$, where the edge-weights $\left(Y_{e}\right)$ are a collection of independent and identically distributed (i.i.d.) random variables. The independence of the edge-weights is a technical assumption that we pose and that makes the analysis of the mathematical model possible, however, we emphasise that it might not hold in real-life applications. The main object of study of first-passage percolation is the time that a flow starting from a vertex $u$ takes to reach a vertex $v$. For an extensive introduction to FPP on random graphs, we refer to [18, Chapter 3].

In this paper, we study first-passage percolation in the setting of the configuration model random graph, with degrees having finite mean and infinite variance. An important question in the study of FPP regards the geometry of the geodesics, or the time-minimizing paths. For this, we consider three functionals on the weighted graph: the graph-distance between two vertices, i.e., the minimal number of edges between them, the weight-distance, i.e., the minimal total weight between the two vertices along all paths connected them, and the hopcount, that is, the number of edges of the minimal-weight path. We focus on the fluctuations of these functionals around their asymptotic mean. We investigate the case of i.i.d. edge-weights of the form $Y=a+X$, where $a$ is a positive constant that we can take to be equal to one without loss of generality and $X$ is a random variable with zero as its infimum of support. In a previous paper [4] we have shown that in a similar setting the weight-distance between two uniformly chosen vertices grows proportionally to $\log \log n$, where $n$ is the number of vertices in the graph. We now extend this result proving that fluctuations around the median are tight for both the weight-distance and the hopcount if and only if the random variable $X$ is such that the continuous-time branching process approximation of the local exploration in the given graph with weight $X$ is explosive. With this we mean that the process has a positive probability of having infinitely many individuals alive in a finite time. This is a non-universality result in contrast with the case of general edge-weights with a continuous distribution and support that contains 0 (see [6]) and finite-variance degrees, in which the hopcount satisfies a central limit theorem. Further, it gives a rather precise picture about the proliferation of almost-shortest paths in the graph distance, and thus about the geometry of the configuration model in the infinite-variance degree setting.

\subsection{Notation and Organization}

In this section we introduce notation used throughout the paper. Given two $\mathbb{R}$-valued random variables $X$ and $Y$, we write $X \stackrel{d}{=} Y$ if $\mathbb{P}(X \leq x)=\mathbb{P}(Y \leq x)$ for all $x \in \mathbb{R}$. With $o_{q}(1)$ we denote a sequence of real numbers such that $o_{q}(1) \rightarrow 0$ as $q \rightarrow \infty$. A sequence of 
random variables $\left(X_{n}\right)_{n \geq 1}$ converges in probability to a random variable $X$, which we write as $X_{n} \stackrel{\mathbb{P}}{\rightarrow} X$ if, for all $\varepsilon>0, \mathbb{P}\left(\left|X_{n}-X\right|>\varepsilon\right) \rightarrow 0$. A sequence of random variables $\left(X_{n}\right)_{n \geq 1}$ converges to $X$ in distribution, which we write as $X_{n} \stackrel{d}{\rightarrow} X$, if $\lim _{n \rightarrow \infty} \mathbb{P}\left(X_{n} \leq\right.$ $x)=\mathbb{P}(X \leq x)$ for all $x \in \mathbb{R}$ for which $F_{X}(x)=\mathbb{P}(X \leq x)$ is continuous. We denote the set $\{1,2, \ldots, n\}$ by $[n]$. A sequence of random variables $\left(X_{n}\right)_{n \geq 1}$ is said to be tight if for all $\varepsilon>0$, there exists $r>0$ such that $\sup _{n \geq 1} \mathbb{P}\left(\left|X_{n}\right|>r\right)<\varepsilon$. A sequence of events $\left(\mathcal{E}_{n}\right)_{n \geq 1}$ is said to hold with high probability (w.h.p.) if $\lim _{n \rightarrow \infty} \mathbb{P}\left(\mathcal{E}_{n}\right)=1$. $\operatorname{Bin}(n, p)$ denotes a random variable with a binomial distribution where the number of trials is $n$ and the success probability is $p . \mathrm{CM}_{n}(\boldsymbol{d})$ denotes the configuration model on $n$ vertices with degree sequence $\boldsymbol{d}=\left(d_{1}, d_{2}, \ldots, d_{n}\right)$, as introduced in the next section.

This section is organized as follows. In Sect. 1.3, we introduce the model. In Sect. 1.4, we describe our results. We close this section in Sect. 1.5 by giving an overview of the proof and relating it to the literature.

\subsection{The Model}

Our setting is the configuration model $\mathrm{CM}_{n}(\boldsymbol{d})$ (see [7]) on $n$ vertices, where $\boldsymbol{d}=$ $\left(d_{1}, \ldots, d_{n}\right)$, and $d_{i}$ is the degree of vertex $i \in[n]$. Further, let $\mathcal{L}_{n}=\sum_{i \in[n]} d_{i}$ denote the total degree. The configuration model graph is obtained as follows: we assign to vertex $i$ a number $d_{i}$ of half-edges and we pair these half-edges uniformly at random. When two halfedges are paired they form an edge, and we continue the procedure until there are no more half-edges available. If $\mathcal{L}_{n}$ is odd, we add a half-edge to vertex $n$; this extra half-edge makes no difference to our results and we will refrain from discussing it further. After the pairing is done, we allocate i.i.d. random variables, that we call edge-weights, from some distribution $Y$, to all existing edges. We consider general degree sequences that asymptotically satisfy a power-law with exponent $\tau \in(2,3)$, so that the variance blows up as $n \rightarrow \infty$. A special case is to consider i.i.d. degrees with cumulative distribution function $x \mapsto F(x)$ satisfying

$$
C x^{-\tau+1} \exp \left\{-C_{1}(\log x)^{\gamma}\right\} \leq 1-F(x) \leq C x^{-\tau+1} \exp \left\{C_{1}(\log x)^{\gamma-1}\right\},
$$

for $\gamma \in(0,1), C, C_{1}>0$ and $\tau \in(2,3)$. We assume that $F(1)=0$, so that $\min _{i \in[n]} d_{i} \geq 2$ a.s. In more general settings, we adapt [20, Assumption 1.1] and impose the following assumption on the empirical degree distribution, $F_{n}(x):=\frac{1}{n} \sum_{v \in[n]} \mathbf{1}_{\left\{d_{v} \leq x\right\}}$ :

Assumption 1.1 (Power-law tail behavior) Fix $\tau \in(2,3)$. There exists $\alpha \in(1 / 2,1 /(\tau-1)]$, $c, C>0$ and $\gamma \in(0,1)$ such that, for all $x$,

$$
1-F_{n}(x) \leq \frac{C}{x^{\tau-1}} \exp \left\{C(\log x)^{\gamma}\right\}
$$

while, for all $x \in\left[x_{0}, n^{\alpha}\right)$,

$$
1-F_{n}(x) \geq \frac{c}{x^{\tau-1}} \exp \left\{-C(\log x)^{\gamma}\right\}
$$

Assumption 1.1 is slightly stronger than [20, Assumption 1.1] in the sense that we deal with appropriate upper and lower bounds on $1-F_{n}(x)$, while [20, Assumption 1.1] also considers truncated degree distributions for which $F_{n}(x)=1$ for all $x \leq n^{\beta_{n}}$ for some $\beta_{n}$ that might or might not tend to zero. Assumption 1.1 forms the main technical assumptions on the degree distribution. Concentration techniques on binomial random variables can be used to prove that Assumption 1.1 holds with high probability in the case of i.i.d. degrees 
whose distribution function satisfies (1.1) (see [19, Section A.1] for details, and in particular $[19,($ A.1.26)]).

The condition on the minimal degree guarantees that almost all the vertices of the graph lie in the same connected component (see [3, Proposition 2.1]), or, equivalently, the giant component has size $n(1-o(1))$ (see [9, Theorem 2.2]). All edges are equipped with i.i.d. edge-weights with distribution function $F_{Y}(y)=\mathbb{P}(Y \leq y)$, where $Y=a+X$ is a nonnegative random variable with $\inf \operatorname{supp}(X)=0, a>0$. Note that we do not assume that $X$ has a continuous distribution. Without loss of generality, we can assume $a=1$. We call $X$ the excess weight of the edge. Let $D_{n}$ have distribution function $F_{n}$, and let $B_{n}$ be defined as the (size-biased version of $D_{n}$ ) -1 , that is,

$$
\mathbb{P}\left(B_{n}=k\right):=\frac{k+1}{\mathbb{E}\left[D_{n}\right]} \mathbb{P}\left(D_{n}=k+1\right) .
$$

We then let $F_{n}^{\star}$ denote the distribution function of $B_{n}$.

In this paper, we consider several distances and functionals on the graph, as defined in the following definition:

Definition 1.2 (Distances in graphs) Given two vertices $u$ and $v$, the graph-distance $\mathcal{D}_{n}(u, v)$ and the passage-time or weight-distance $W_{n}(u, v)$ from $u$ to $v$ are defined as

$$
\mathcal{D}_{n}(u, v):=\min _{\pi: u \rightarrow v} \sum_{e \in \pi} 1, \quad W_{n}(u, v):=W_{n}^{Y}(u, v)=\min _{\pi: u \rightarrow v} \sum_{e \in \pi} Y_{e},
$$

where the minimum is over all paths $\pi$ in $G$ connecting $u$ and $v$, and $Y:=\left(Y_{e}\right)_{e}$ is the collection of edge-weights. The hopcount $H_{n}(u, v)$ is the number of edges in the minimal weight path between $u$ and $v$ (if this path is non-unique, we take the one with minimal number of edges on it).

The main aim of this paper is to study these functionals on the configuration model with degrees having empirical distribution function $F_{n}$ satisfying (1.1) and i.i.d. edge-weights $Y=1+X$, where inf $\operatorname{supp}(X)=0$.

\subsection{Results}

Our main result is the following theorem:

Theorem 1.3 (Tightness criterion for excess edge-weights) Consider $\mathrm{CM}_{n}(\boldsymbol{d})$, where the degrees satisfy Assumption 1.1 for some $\tau \in(2,3)$, and assume that $F_{n}(1)=0$. Let $u$ and $v$ be chosen uniformly at random from $[n]$. Suppose that the edge-weights are i.i.d. and are of the form $Y=1+X$, where $\inf \operatorname{supp}(X)=0$ and $X$ has cumulative distribution function $F_{X}(x)$ with its generalised inverse $F_{X}^{(-1)}(y):=\inf \left\{t \in \mathbb{R}: F_{X}(t) \geq y\right\}$ that satisfies that, for some $\varepsilon>0$,

$$
\mathbf{I}(X):=\int_{1 / \varepsilon}^{\infty} F_{X}^{(-1)}\left(\mathrm{e}^{-u}\right) \frac{1}{u} \mathrm{~d} u<\infty .
$$

Then

$$
W_{n}(u, v)-\frac{2 \log \log n}{|\log (\tau-2)|}
$$


is a tight sequence of random variables. Consequently, also

$$
H_{n}(u, v)-\frac{2 \log \log n}{|\log (\tau-2)|}
$$

is a tight sequence of random variables.

The tightness of the hopcount in (1.8) is a rather direct consequence of a similar tightness result for the typical distance $\mathcal{D}_{n}(u, v)$, since, for our choice of edge-weights,

$$
\mathcal{D}_{n}(u, v) \leq H_{n}(u, v) \leq W_{n}(u, v) .
$$

Therefore, by (1.7) in Theorem 1.3 and the tightness of

$$
\mathcal{D}_{n}(u, v)-\frac{2 \log \log n}{|\log (\tau-2)|}
$$

as proved in [19, Theorem 1.2] in the setting of i.i.d. degrees, it follows that (1.8) holds. In the setting of general degrees under Assumption 1.1, we prove this tightness in Proposition 1.8 below. Tightness of the sequence in (1.10) also follows from [20], under Assumption 1.1.

Remark 1.4 The integral condition in (1.6) is equivalent to the following. Let $B_{n}$ be defined in (1.4) and $X$ be the excess edge-weight. Assume that $B_{n} \stackrel{d}{\longrightarrow} B$ for some random variable $B$. Let $\mathrm{BP}(B, X)$ be the age-dependent branching process where individuals have random lifelengths with distribution $X$. At death, every individual, independently, produces a family of random size with offspring distribution $B$. Then, (1.6) holds if and only if this age-dependent branching process is explosive, meaning that there is a positive probability that $N_{t}=\infty$, where $N_{t}$ denotes the number of individuals alive at some finite time $t>0$. Otherwise, the process is called conservative. See [2] or [14, Section 6] for the proof of this result. Note in particular that this property is independent of the precise value of the power-law exponent $\tau \in(2,3)$ in (1.1) (that enters the age-dependent branching process through (1.4)). We emphasise this connection below in Theorem 1.10 below.

Remark 1.5 We emphasize that the integral condition in (1.6) is a criterion that is easy to verify for most commonly known distributions. The convergence of the integral depends on the behavior of the distribution of $X$ around 0 : the steeper the distribution function at 0 , the smaller the lhs of (1.6). For example, (1.6) holds for Exponential, Uniform, Gamma distributions, etc, but also distributions that are more flat around the origin. The boundary case is when $F_{X}$ is doubly-exponentially flat around 0 , i.e., the family of distributions $F_{X}(t)=$ $c_{1} \exp \left\{-c_{2} \exp \left\{c_{3} / t^{\beta}\right\}\right\}$, for appropriate constants $c_{1}, c_{2}, c_{3}>0$ and parameter $\beta$. For this case, (1.6) is satisfied for $\beta<1$ but the integral diverges for $\beta \geq 1$. We remark that $F_{X}(x)$ does not have to be continuous to satisfy (1.6). For instance, a distribution that puts point masses $c_{1}^{k} /\left(1-c_{1}\right)$ to location $c_{2}^{k}$, for all $k \geq 0$ can be shown to satisfy (1.6) for all $c_{1}, c_{2} \in(0,1)$. Finally, we remark that it is allowed that $\mathbb{P}(X=\infty)>0$. In this case one just needs to set $1 / \varepsilon$ in the integration boundary (1.6) sufficiently high so that the inverse is defined.

We next investigate what happens when the criterion in (1.6) fails:

Proposition 1.6 (Non-tightness of excess edge-weight) Consider $\mathrm{CM}_{n}(\boldsymbol{d})$, where the degree sequence satisfies Assumption 1.1 for some $\tau \in(2,3)$, and assume that $F_{n}(1)=0$. Let $u$ and $v$ be chosen uniformly at random from $[n]$. Suppose that the edge-weights are i.i.d. and are 
of the form $Y=1+X$, where $\inf \operatorname{supp}(X)=0$ and $X$ has cumulative distribution function $F_{X}(x)$ that satisfies for some $\varepsilon>0$ that $\mathbf{I}(X)=\infty$ in (1.6). Then

$$
W_{n}(u, v)-\frac{2 \log \log n}{|\log (\tau-2)|} \stackrel{\mathbb{P}}{\longrightarrow} \infty .
$$

Further, also $W_{n}(u, v)-H_{n}(u, v) \stackrel{\mathbb{P}}{\longrightarrow} \infty$.

To prove Proposition 1.6, we state and prove a useful lemma:

Lemma 1.7 Consider $\mathrm{CM}_{n}(\boldsymbol{d})$, where the degrees satisfy Assumption 1.1 for some $\tau \in(2,3)$, and assume that $F_{n}(1)=0$. Let $u$ and $v$ be chosen uniformly from $[n]$. Suppose that the edge-weights are i.i.d. and are of the form $X$, where inf $\operatorname{supp}(X)=0$ and $X$ has cumulative distribution function $F_{X}(x)$ that satisfies for some $\varepsilon>0$ that $\mathbf{I}(X)=\infty$ in (1.6). Then

$$
W_{n}^{X}(u, v) \stackrel{\mathbb{P}}{\longrightarrow} \infty \text {. }
$$

Proof The statement is a consequence of the branching process approximation of the neighborhood of a vertex in $\mathrm{CM}_{n}(\boldsymbol{d})$ and the hypothesis that the process is not explosive, see [4] and [14, (11) and Proposition 3] in the context of i.i.d. degrees, and [1] for general degrees. In more detail, [1] shows in this setting the stronger result

$$
W_{n}^{X}(u, v) / \sum_{k=1}^{\log \log n /|\log (\tau-2)|} F_{X}^{(-1)}\left(\exp \left(-(\tau-2)^{-k}\right)\right) \stackrel{\mathbb{P}}{\longrightarrow} 1 .
$$

The proof of Proposition 1.6 given Lemma 1.7 is as follows:

Proof of Proposition 1.6 Let $Y_{e}=X_{e}+Z_{e}$, where $Y_{e}, X_{e}$ and $Z_{e}$ are edge-weights. For a path $\pi$, write $W_{n}^{Z}(\pi)=\sum_{e \in \pi} Z_{e}$ (and the same for $X, Y$ ). Then, for any pair of vertices $u$ and $v$ in the same connected component and any path $\pi$ connecting $u$ and $v$,

$$
W_{n}^{Y}(\pi)=W_{n}^{X}(\pi)+W_{n}^{Z}(\pi) \geq W_{n}^{X}(u, v)+W_{n}^{Z}(\pi) .
$$

Let $\pi^{Y}$ be the minimal-weight path between $u$ and $v$ for the weights $\left(Y_{e}\right)_{e}$ and let $Z_{e} \equiv 1$, so that $W_{n}^{Y}\left(\pi^{Y}\right)=W_{n}^{Y}(u, v)$, while $W_{n}^{Z}\left(\pi^{Y}\right)=H_{n}(u, v)$. Then, we can rewrite (1.13) as

$$
W_{n}^{Y}(u, v)-H_{n}(u, v) \geq W_{n}^{X}(u, v) .
$$

If $X_{e}$ is such that $\mathbf{I}(X)=\infty$, then, by Lemma $1.7, W_{n}^{X}(u, v) \stackrel{\mathbb{P}}{\longrightarrow} \infty$, which implies that $W_{n}^{Y}(u, v)-H_{n}(u, v) \stackrel{\mathbb{P}}{\longrightarrow} \infty$, as required. For the statement that $W_{n}(u, v)-$ $2 \log \log n /|\log (\tau-2)| \stackrel{\mathbb{P}}{\longrightarrow} \infty$, we note that $H_{n}(u, v) \geq \mathcal{D}_{n}(u, v)$, so that $W_{n}^{Y}(u, v)-\frac{2 \log \log n}{|\log (\tau-2)|} \geq\left[W_{n}^{Y}(u, v)-H_{n}(u, v)\right]+\left[\mathcal{D}_{n}(u, v)-\frac{2 \log \log n}{|\log (\tau-2)|}\right] \stackrel{\mathbb{P}}{\longrightarrow} \infty$,

since $\mathcal{D}_{n}(u, v)-2 \log \log n /|\log (\tau-2)|$ is a tight sequence of random variables by [20].

Proposition 1.6 already highlights the importance of tightness of graph distances in our setting. We next show that the difference between the graph-distance and $2 \log \log n / \mid \log (\tau-$ 2)| is tight: 
Proposition 1.8 (Lower tightness of graph distances) Consider $\mathrm{CM}_{n}(\boldsymbol{d})$ where the degree sequence $\boldsymbol{d}=\left(d_{1}, \ldots, d_{n}\right)$ has empirical distribution function $F_{n}(x)$. Suppose that there exist $C>0, C_{1} \in \mathbb{R}, \gamma \in(0,1)$ such that

$$
1-F_{n}(x) \leq \frac{C}{x^{\tau-1}} \exp \left\{C_{1}(\log x)^{\gamma}\right\} \quad \text { for all } x \geq 1 .
$$

Then, $\mathcal{D}_{n}(u, v)-2 \log \log n /|\log (\tau-2)|$ satisfies

$$
\lim _{K \rightarrow \infty} \sup _{n \geq 1} \mathbb{P}\left(\mathcal{D}_{n}(u, v)-\frac{2 \log \log n}{|\log (\tau-2)|} \leq-K\right)=0 .
$$

Remark 1.9 (I.i.d. vs. non-i.i.d. degrees) For the i.i.d. case satisfying (1.1), the tightness of $\mathcal{D}_{n}(u, v)-2 \log \log n /|\log (\tau-2)|$ has already been proved in [19]. Slightly sharper conditions have appeared in the branching process context by Davies in [8], who investigates the number of particles in an infinite-mean (Galton-Watson) branching process. It is unclear whether the result also holds when $\exp \left\{C_{1}(\log x)^{\gamma}\right\}$ is replaced by an arbitrary slowly-varying function. We will use the result by Davies [8] in the proof of Proposition 1.8 below.

We now prove Proposition 1.8 and Lemma 1.7, using a branching process comparison:

Proof (Sketch of proof of Proposition 1.8 and Lemma 1.7) We only provide the sketch of one possible proof, since many similar results are proved in the literature and we refer to some of them. Let $F_{n}^{\star}$ denote the distribution function of $B_{n}$ in (1.4). When (1.16) holds, also

$$
\begin{aligned}
1-F_{n}^{\star}(x) & =\sum_{k>x} \frac{k+1}{\mathbb{E}\left[D_{n}\right]} \mathbb{P}\left(D_{n}=k+1\right) \\
& =\frac{x}{\mathbb{E}\left[D_{n}\right]}\left[1-F_{n}\right](x)+\frac{1}{\mathbb{E}\left[D_{n}\right]} \sum_{k>x}\left[1-F_{n}\right](k) \\
& \leq \frac{C^{\star}}{x^{\tau-2}} \exp \left\{C_{1}^{\star}(\log x)^{\gamma}\right\}
\end{aligned}
$$

for an appropriately chosen constants $C^{\star}, C_{1}^{\star}$. See [20,(1.7)] where such an estimate is worked out in more detail. We explore the neighborhoods of $u$ and $v$ in a breadth-first way, and denote the arising forward degrees by $\left(\widetilde{B}_{n, i}^{(u)}\right)_{i \geq 1}$ and $\left(\widetilde{B}_{n, i}^{(v)}\right)_{i \geq 1}$ respectively, and the degrees of the uniform vertices by $\widetilde{D}_{n}^{(u)}=d_{u}$ and $\widetilde{D}_{n}^{(v)}=d_{v}$. By a standard coupling argument as, for example, worked out in detail in [18, Lemma 2.12], we can couple $\left(\widetilde{D}_{n}^{(u)},\left(\widetilde{B}_{n, i}^{(u)}\right)_{i \leq m_{n}}, \widetilde{D}_{n}^{(v)},\left(\widetilde{B}_{n, i}^{(v)}\right)_{i \leq m_{n}}\right)$ to an independent sequence $\left(D_{n}^{(u)},\left(B_{n, i}^{(u)}\right)_{i \leq m_{n}}, D_{n}^{(v)},\left(B_{n, i}^{(v)}\right)_{i \leq m_{n}}\right)$, where $D_{n}^{(u)}, D_{n}^{(v)}$ have distribution $D_{n}$ and $B_{n, i}^{(u)}, B_{n, i}^{(v)}$ distribution $B_{n}$ in (1.4), as long as $m_{n}=o\left(\sqrt{n / d_{\max }}\right)$, where $d_{\max }$ is the maximal degree. When (1.16) holds, $d_{\max }=O\left(n^{1 /(\tau-1)}\right)$, so this successful coupling applies as long as $m_{n}=n^{\kappa}$ for any $\kappa<(\tau-2) /[2(\tau-1)]$. In particular, this implies that the two neighborhoods of $u$ and $v$ are w.h.p. disjoint up to the moment that each contains $m_{n}$ vertices.

Further, when (1.16) holds, and using (1.18), the random variables $D_{n}$ and $B_{n}$ can be stochastically bounded from above by random variables $\bar{D}$ and $\bar{B}$ with distribution functions $F_{\bar{D}}(x)=1-C x^{-(\tau-2)} \exp \left\{C_{1}(\log x)^{\gamma-1}\right\}$ and $F_{\bar{B}}(x)=1-C^{\star} x^{-(\tau-2)} \exp \left\{C_{1}^{\star}(\log x)^{\gamma-1}\right\}$, respectively. In this setting, we can apply the branching process result by Davies [8], showing that $\bar{Z}_{k}$, the number of individuals in generation $k$ in the two-stage branching process with root offspring distribution $\bar{D}$ and offspring distribution $\bar{B}$ in any other generation, satisfies

$$
(\tau-2)^{k} \log \left(\bar{Z}_{k}\right) \stackrel{\text { a.s. }}{\longrightarrow} \bar{V},
$$


for some non-negative random variable $\bar{Y}$. Solving $\bar{Z}_{k} \leq \exp \left\{(\tau-2)^{-k}(\bar{V}+\varepsilon)\right\} \leq m_{n}=$ $n^{\kappa}$ for $k$ yields $k \leq\left(\log \log n-\log \left(\left(\bar{V}_{u}+\varepsilon\right) / \kappa\right)\right) /|\log (\tau-2)|$ w.h.p. This implies that the neighborhood of $u$ of size $m_{n}$ vertices w.h.p. contain the first $\left(\log \log n-\log \left(\left(\bar{V}_{u}+\right.\right.\right.$ $\varepsilon) / \kappa)) /|\log (\tau-2)|$ generations, where $\bar{V}_{u}$ is the random variable $\bar{V}$ corresponding to the two-stage branching process rooted at $u$. The same applies to $v$. Thus, since the two clusters are disjoint w.h.p.,

$$
\begin{aligned}
\mathcal{D}_{n}(u, v) \geq & \left(\log \log n-\log \left(\left(\bar{V}_{u}+\varepsilon\right) / \kappa\right)\right) /|\log (\tau-2)| \\
& +\left(\log \log n-\log \left(\left(\bar{V}_{v}+\varepsilon\right) / \kappa\right)\right) /|\log (\tau-2)| \\
= & \left.2 \log \log n /|\log (\tau-2)|-\log \left(\left(\bar{V}_{u}+\varepsilon\right)\left(\bar{V}_{v}+\varepsilon\right) / \kappa^{2}\right)\right) /|\log (\tau-2)|,
\end{aligned}
$$

as required. Since the last term is w.h.p. $\geq-K$ when $K$ is large, the claim follows. This completes the proof of Proposition 1.8.

The proof of Lemma 1.7 makes use of the same coupling, but now note that when condition (1.6) fails, then the continuous-time branching process with edge-weights $X$ and offspring distribution $\bar{B}$ is not explosive, so that the time to leave the $m_{n}$-environment of $u$ tends to infinity almost surely.

We close this results section by merging Proposition 1.6 and Theorem 1.3 in a single theorem for i.i.d. degrees. For this, let $F_{X}$ be the cumulative distribution for the random variable $X$, let $D$ be the degree distribution and $B$ the random variable defined in (1.4), with $D_{n}$ replaced by $D$. If $\mathrm{BP}(B, X)$ is the age-dependent branching process defined with offspring distribution $B$ and life-length distribution $X$ (see Remark 1.4), then we can define the following sets:

$$
\mathcal{E}(D)=\left\{F_{X}: \mathrm{BP}(B, X) \text { is explosive }\right\}
$$

and

$$
\mathcal{T}(D)=\left\{F_{X}: W_{n}^{(1+X)}-\frac{2 \log \log n}{|\log (\tau-2)|} \text { is tight }\right\} .
$$

Theorem 1.10 (Universality class for tightness = universality class for explosion) Consider $\mathrm{CM}_{n}(\boldsymbol{d})$, where the degrees are i.i.d. from some distribution $D$ that satisfies (1.1) for some $\tau \in(2,3)$, and assume that $F_{D}(1)=0$. Then

$$
\mathcal{E}(D)=\mathcal{T}(D) \text {. }
$$

Proof The proof follows directly from Proposition 1.6, Theorem 1.3 and Remark 1.4.

Remark 1.11 (Universality and proliferation of almost-shortest paths) Proposition 1.6 shows that there are two universality classes in terms of tightness for the case where the edge-weights take the form $Y=1+X$ where $\inf \operatorname{supp}(X)=0$. Namely, one where (1.6) is satisfied and one where it is not. The same is true for typical distances in the setting of edge-weights $\left(Y_{e}\right)_{e}$ with $\inf \operatorname{supp}(Y)=0$ (see [4, Theorem 4]). In that case, (1.6) implies distributional convergence of typical distances, while divergence of the integral in (1.6) implies typical distances tending to infinity. Remarkably, Theorem 1.10 shows that these two universality classes are the same.

The proof of Theorem 1.3 also shows that there are many almost-shortest paths in the graph-distance metric, since we can even find one with tight total excess edge-weight when (1.6) is satisfied. However, when the excess edge-weight distribution increases too thinly 
from zero, such that (1.6) fails, such paths can no longer be found. This gives us a more complete picture of the geometry of the configuration model with infinite-variance degrees, and brings the discussion of the universality classes of FPP on it substantially further.

Theorems 1.3 and 1.10 leave open whether the fluctuations converge in distribution. That is part of the following open problem:

Open Problem 1.12 (Weak convergence of fluctuations) Consider $\mathrm{CM}_{n}(\boldsymbol{d})$, where the degrees are i.i.d. from distribution $D$ that satisfies sequence satisfies (1.1) with $\tau \in(2,3)$, and assume that $F_{n}(1)=0$. Let $u$ and $v$ be chosen uniformly at random from $[n]$. Suppose that the edge-weights are i.i.d. and are of the form $Y=1+X$, where inf $\operatorname{supp}(X)=0$ and $X$ has cumulative distribution function $F_{X}(x)$. Show that when $\mathbf{I}(X)<\infty$ in (1.6) for some $\varepsilon>0$, then

$$
W_{n}^{Y}(u, v)-\frac{2 \log \log n}{|\log (\tau-2)|} \stackrel{d}{\longrightarrow} W_{\infty}
$$

for some limiting random variable $W_{\infty}$. When $\mathbf{I}(X)=\infty$, is then

$$
W_{n}^{Y}(u, v)-\frac{2 \log \log n}{|\log (\tau-2)|}
$$

of the same order of magnitude as $W_{n}^{X}(u, v)$ ?

In Open Problem 1.12, we resort to i.i.d. degrees, as the limiting distribution $W_{\infty}$ can be expected to depend sensitively on the precise degree distribution (not just upper and lower bounds on its empirical distribution function as in Assumption 1.1).

\subsection{Overview of the Proof}

\subsubsection{Upper Tightness of the Graph-Distance $\mathcal{D}_{n}(u, v)$}

First we prove a uniform upper bound on the difference between the graph-distance of two vertices of sufficiently high degree (at least $k$ ) and $2 \log \log n /|\log (\tau-2)|$. For this we construct a path that has length less than $2 \log \log n / \log (\tau-2) \mid$ plus a tight random variable. The construction is as follows: we start from vertex $u_{k}$ with degree at least $k$, for a fixed but large constant $k$. Then we find a sequence of nested sets $\Gamma_{y_{i}}: \Gamma_{y_{i}} \supset \Gamma_{y_{i+1}}$, where $0 \leq i \leq b, \Gamma_{y_{i}}=\left\{v: d_{v} \geq y_{i}\right\}$, and $b$ is less than $\log \log n /|\log (\tau-2)|$, for some increasing sequence $y_{i}$. We show that for any fixed small $\varepsilon>0$ there exists an increasing sequence $y_{i}$ such that the following properties hold: $y_{0}=k$ and a vertex in $\Gamma_{y_{i}}$ is connected to at least one vertex in $\Gamma_{y_{i+1}}$ with probability at least $1-\varepsilon_{i}$, and $\sum_{i=0}^{b} \varepsilon_{i}<\varepsilon$. Here, the path is constructed sequentially, and the vertex that is found in $\Gamma_{y_{i}}$ is then w.h.p. connected to at least one vertex in $\Gamma_{y_{i+1}}$. Moreover, the last set of the sequence $\Gamma_{y_{b}}$ is a subset of the complete graph formed by hubs, by which we mean degree at least $n^{1 / 2+\delta}$ for some small $\delta>0$. We show that the number of sets needed, $b$, is at most $\log \log n /|\log (\tau-2)|$ when $k$ is sufficiently large. So, starting from two uniformly chosen vertices $u_{k}$ and $v_{k}$ with degree at least $k$, the two paths constructed with the procedure above, we connect $u_{k}$ and $v_{k}$ to the same complete graph in a number of steps that is at most $2 \log \log n /|\log (\tau-2)|$. 


\subsubsection{Degree-Dependent Percolation on Configuration Models}

Our proof of the upper tightness in (1.7) crucially relies on a percolation argument that we believe to be of independent interest. We call this degree-dependent percolation. In this percolation argument, we independently keep or remove each half-edge $s$ with probability $p(d)$ vs. $1-p(d)$, where $d$ is the degree of the vertex that $s$ is attached to. Then, each edge is removed when at least one of the half-edges of which it consists is removed. We show that a giant component remains when $p(d) \geq \exp \left\{-C \log (d)^{\gamma}\right\}$, and that the typical distances $\mathcal{D}_{n}^{r}(u, v)$ within the giant component remaining after percolation still satisfy that $\mathcal{D}_{n}^{r}(u, v)-2 \log \log n /|\log (\tau-2)|$ is tight. This is achieved by relating the degree-percolated graph to another configuration model, for which the degrees w.h.p. still satisfy the lower bound in (1.3) of Assumption 1.1, with the same value of $\tau \in(2,3)$. For this, we extend the construction by Janson (see [12]) to this degree-dependent percolation on the configuration model.

\subsubsection{Tightness of the Weight-Distance $W_{n}^{Y}(u, v)$ via Degree-Dependent Percolation}

To apply the above degree-dependent percolation result, we choose $p(d)$ in such a way that it can be expressed as $\mathbb{P}\left(X \leq \xi_{d}\right)$, for some appropriately chosen sequence $\xi_{d}$ that depends on the distribution of the excess edge-weight $X$. Then, we can use the (independent) extra edge-weights $\left(X_{e}\right)_{e}$ as auxiliary random variables to decide which edge to keep or remove: we keep a half-edge $s$ attached to a vertex with degree $d$ if and only if the excess edge-weight on the half-edge $s$ satisfies $X_{s} \leq \xi_{d}$. Note that in Theorem 1.3, we have assumed that the weight on an edge is of the form $Y=1+X$, while the degree-dependent percolation assigns a weight to each half-edge. To solve this issue, we solve the case when the edge-weights are of the form $Y^{\prime}=1+X_{1}+X_{2}$ where $X_{1}$ and $X_{2}$ are two i.i.d. random variables from distribution $X$. Since $1+X \stackrel{d}{\leq} 1+X_{1}+X_{2}$, weight-distances in the graph with edgeweights $Y$ are stochastically smaller than weight-distances in the graph with edge-weights $Y^{\prime}$. Further, both distances are bounded from below by the graph-distance. Hence, tightness of the weight-distance with respect to the edge-weights from distribution $Y^{\prime}$ implies tightness of the weight-distance with respect to the edge-weights from distribution $Y$. This is reflected in the fact that the integral criterion for $X$ and $X_{1}+X_{2}$ in (1.6) can be seen to agree.

Starting from $\mathrm{CM}_{n}(\boldsymbol{d})$, where the degrees satisfy Assumption 1.1 for some $\tau \in(2,3)$, we prove that under the condition that (1.6) is satisfied, it is possible to choose $\xi_{d}$ and thus $p(d)$ in such a way that the new percolated graph has a new empirical degree distribution that still satisfies the lower bound in (1.3) in Assumption 1.1 with the same exponent $\tau$. This implies that the setting that allows the construction of the path described in Sect. 1.5.1 is maintained in the percolated graph. Namely, to construct a path from $u$ to $v$ with bounded excess edge-weight, we use two steps. First, we fix some large constant $k$ and we approximate a constant-size neighborhood of the vertices $u, v$ in the original, unpercolated graph by two branching processes, in order to reach two vertices $u_{k}, v_{k}$ that have degree at least $k$ after percolation w.h.p. Then, in the second step, we connect $u_{k}$ to $v_{k}$ within the percolated graph with a bounded excess edge-weight by constructing a path $\pi^{\star}$. Thus,

$$
W_{n}(u, v) \leq W_{n}\left(u, u_{k}\right)+W_{n}\left(v, v_{k}\right)+\left|\pi^{\star}\right|+\sum_{x \in \pi^{*}} 2 \xi_{d_{x}},
$$

where $\left|\pi^{\star}\right|$ is the number of edges on the constructed path and $\xi_{d_{x}}$ is the upper bound on the excess edge-weight on the half-edges that are attached to vertex $x$ in the percolated graph. 
The first two terms are tight since $k$ is fixed, the third one is $2 \log \log n /|\log (\tau-2)|+$ a $k$-dependent constant, by construction, while the final term can be seen to be tight for an appropriate choice of the $\left(\xi_{d}\right)$ precisely when (1.6) is satisfied. We mention that by choosing $k$ large enough, we also ensure that $u_{k}, v_{k}$ belong to the giant component of the percolated graph w.h.p., i.e., the path $\pi^{\star}$ can be constructed. This describes the structure of the proof.

While we see that explosion of FPP with edge-weights $\left(X_{e}\right)_{e}$ occurs if and only if $W_{n}^{1+X}(u, v)-2 \log \log n /|\log (\tau-2)|$ is tight (recall Theorem 1.10), we have no heuristic idea for one of the directions. The fact that non-explosion implies non-tightness has an intuitive proof, see Proposition 1.6. The other direction, however, uses the complicated degree-dependent percolation argument. It would be of interest to understand the heuristics behind that direction better.

\subsection{Discussion and Related Problems}

First-passage percolation has been studied extensively in different settings, starting from the grid $\mathbb{Z}^{2}$ to a wide variety of random graphs, including the configuration model. One of the main problems in first-passage percolation regards the typical weight-distance between two vertices in the graph. Moreover, if we assume that the edges have a passage-time represented by a collections of i.i.d. random variables, a second problem is to determine the geometry of the time-minimizing paths between two points and the way in which they differ from graphdistance paths. A third problem regards the nature of the fluctuations of these distances and of the hopcount around their asymptotic mean values.

In the context of random and complete graphs, these questions have been widely investigated, for instance in [11], Janson proves that on the complete graph $K_{n}$ with i.i.d. exponential weights, the weight-distance between two points grows asymptotically as $\log n / n$. Bhamidi, the second author and Hooghiemstra in [6] examine the Erdôs-Rényi random graph $G_{n}\left(p_{n}\right)$ with i.i.d. exponential edge-weights. When $n p_{n} \rightarrow \lambda>1$, the weight-distance centered by a multiple of $\log n$ converges in distribution, while, when $n p_{n} \rightarrow \infty$ they prove that the graphdistance is of order $o(\log n)$, and that the addition of edge-weights changes the geometry of the graph. The same authors show in [5] that on the configuration model, when the degree sequence has finite variance with an extra logarithmic moment, first-passage percolation has only one universality class in the sense that $W_{n}(u, v)-\gamma_{n} \log n$ converges in distribution for some $\gamma_{n} \rightarrow \gamma>0$, while $H_{n}(u, v)$ satisfies an asymptotic Central Limit Theorem with asymptotic mean and variance proportional to $\log n$. In [16], van den Esker, the second author and Hooghiemstra generalize the results on configuration model with finite-variance degrees to a more general class of random graphs, including the Erdős-Rényi random graph, showing that the fluctuations around the asymptotic mean are tight.

The setting of configuration models with power-law degrees having infinite asymptotic variance has also been investigated. However, here the picture is less complete: In [4], we prove results for the weight-distance for i.i.d. edge-weights $X$ with inf $\operatorname{supp}(X)=0$. We have shown the existence of two universality classes, one corresponding to explosive weights as in (1.21) and one corresponding to conservative weights. These two classes correspond to different weight-distances, in particular, in the explosive case, the weight converges to the sum of two i.i.d. random variables. Adriaans and the third author [1] investigate the leading order behavior of the weight-distance in the conservative case. A result on the nature of fluctuation is given by the second author, Hooghiemstra and Znamenski in [19], where they proved that the graph-distance for power-law exponent $\tau \in(2,3)$ and i.i.d. degrees centers around $2 \log \log n /|\log (\tau-2)|$, and that the fluctuations are tight. In a recent paper, the 
second and third author generalized these results by investigating the fluctuation of graph distances when the degrees are truncated at a lower value $n^{\beta_{n}}$ rather than $n^{1 /(\tau-1)}$, which is the maximal degree for i.i.d. degrees, see [20]. We refer to [18, Chapter 3] for an extensive discussion of results for FPP on random graphs.

Organization of this paper In Sect. 2 we prove upper tightness of $\mathcal{D}_{n}(u, v)-$ $2 \log \log n /|\log (\tau-2)|$ under relatively weak assumptions on the degrees, which is a crucial ingredient in our proof. In Sect. 3 we combine this result with a degree-dependent half-edge percolation argument that enables us to find the path $\pi^{\star}$ in the percolated graph. In Sect. 4 we complete the proof of tightness of the weight-distance.

\section{Tightness of the Graph-Distance}

In this section, we prove the upper tightness result on $\mathcal{D}_{n}(u, v)-2 \log \log n /|\log (\tau-2)|$ that complements the lower tightness result in Proposition 1.8. We further prove additional properties depending on the degree of the vertices $u, v$ as well as on the degrees of the vertices in the connecting path, which will be crucial in order to apply the result in the context of FPP on $\mathrm{CM}_{n}(\boldsymbol{d})$. With $\mathcal{D}_{n}(u, v):=\infty$ if $u, v$ are not connected, we have the following proposition:

Proposition 2.1 (Upper tightness of graph distances) Consider $\mathrm{CM}_{n}(\boldsymbol{d})$ with degree sequence $\boldsymbol{d}=\left(d_{1}, \ldots, d_{n}\right)$ with empirical distribution function $F_{n}(x)$. Suppose that there exist $\alpha>$ $1 / 2, \beta, c>0, C_{1} \in \mathbb{R}$ and $\gamma \in(0,1)$ such that

$$
1-F_{n}(x) \geq \frac{c}{x^{\tau-1}} \exp \left\{-C_{1}(\log x)^{\gamma}\right\} \quad \text { for all } x \in\left[1, n^{\alpha}\right),
$$

with $\mathcal{L}_{n}=\sum_{i \in[n]} d_{i} \leq n \beta$. Then, the following holds:

(a) Let us set $b(n)=\log \log n /|\log (\tau-2)|$, and introduce the event

$$
\begin{aligned}
\mathcal{E}_{n}:=\left\{\exists b \leq b(n), \text { and a path }\left(u=\pi_{0}, \pi_{1}, \ldots, \pi_{2 b+1}=v\right)\right. \text { with } \\
\left.d_{\pi_{i}} \geq\left(k^{1-\delta}\right)^{(\tau-2)^{-i \wedge(2 b+1-i)}}\right\} .
\end{aligned}
$$

For all $\varepsilon_{2.1}>0$, there exists $k=k\left(\varepsilon_{2.1}\right) \in \mathbb{N}$ and $n=n\left(\varepsilon_{2.1}\right)$, s.t., for all $n \geq n\left(\varepsilon_{2.1}\right)$, when $u$ and $v$ are two uniformly chosen vertices in $[n]$,

$$
\mathbb{P}\left(\mathcal{E}_{n}^{c} \mid d_{u} \geq k, d_{v} \geq k\right)<\varepsilon_{2.1} .
$$

Thus, since $\mathcal{E}_{n} \subset\left\{\mathcal{D}_{n}(u, v)-2 \log \log n /|\log (\tau-2)| \leq 1\right\}$, we obtain

$$
\mathbb{P}\left(\mathcal{D}_{n}(u, v)-\frac{2 \log \log n}{|\log (\tau-2)|}>1 \mid d_{u} \geq k, d_{v} \geq k\right)<\varepsilon_{2.1} .
$$

(b) The same results hold conditionally on $d_{u}=\ell_{1}, d_{v}=\ell_{2}$ whenever $\ell_{1}, \ell_{2} \geq k$, with error bound $\varepsilon_{2.1}$ from (2.3) valid uniformly for all $\ell_{1}, \ell_{2} \geq k$.

Note that (2.4) includes the estimate on the probability of the event that $\mathcal{D}_{n}(u, v)=\infty$, i.e., when $u, v$ are not connected. To prove Proposition 2.1 we build a connecting path $\pi$ using a nested sequence of layers in $\mathrm{CM}_{n}(\boldsymbol{d})$ of the form $\Gamma_{y_{i}}$, where for a positive number $y$, we let $\Gamma_{y}:=\left\{v: d_{v} \geq y\right\}$. Let

$$
\Lambda_{\eta}=\left\{v: d_{v} \geq n^{\eta}\right\}=\Gamma_{n^{\eta}}
$$


Our aim is to prove that for any small $\varepsilon>0$, there exists an increasing sequence $\left(y_{i}\right)_{i \leq b}$, with $y_{0}=k$ s.t. $\left(\Gamma_{y_{i}}\right)_{i \leq b}$ has the following properties:

1. $u=\pi_{0} \in \Gamma_{y_{0}}$ chosen uniformly in $\left\{x \in[n]: d_{x} \geq k\right\}$. With probability at least $1-\varepsilon$, there exists a sequence $\left(\pi_{i}\right)_{i \leq b}$ with $b \leq b(n)$, such that $\pi_{i}$ is connected to $\pi_{i+1}$, and $\pi_{i} \in \Gamma_{y_{i}}$ is chosen in $\Gamma_{y_{i}}$ according to the size-biased distribution in $\Gamma_{y_{i}}$, for all $i \geq 1$.

2. $\pi_{b}$ is w.h.p. connected to the set $\Lambda_{\eta}$ for some $\eta>1 / 2$ by an edge ${ }^{1}$.

For this, we choose a sequence $\left(y_{i}\right)_{i \geq 0}$ with $y_{0}=k$ that satisfies the following: Let $\pi_{0}=u$, and define $\left(\pi_{i}\right)_{i \geq 0}$ recursively as follows. Let $\pi_{i+1}$ be the vertex in $\Gamma_{y_{i+1}}$ that is adjacent to the half-edge paired to the first half-edge attached to $\pi_{i}$ that is connected to a half-edge incident to $\Gamma_{y_{i+1}}$ (if this exists). Since the half-edge attached to $\pi_{i+1}$ that forms the edge $\left(\pi_{i}, \pi_{i+1}\right)$ is chosen uniformly among the half-edges incident to $\Gamma_{y_{i+1}}, \pi_{i+1}$ is a vertex chosen according to the size-biased distribution in $\Gamma_{y_{i+1}}$ for all $i \geq 0$. Let $E_{i}:=$ $\left\{\pi_{i}\right.$ is not connected to $\left.\Gamma_{y_{i+1}}\right\}=\left\{\pi_{i+1}\right.$ does not exist $\}$. Since $d_{\pi_{i}} \geq y_{i}$, the bounds on $d_{\pi_{i}}$ stated in Proposition 2.1 will follow directly from the choice of $y_{i}$ (see also Lemma 2.4 below, where the precise bound is proved). We want to prove show that for all $k$ sufficiently large,

$$
\sum_{i=0}^{b} \mathbb{P}\left(\pi_{i} \in \Gamma_{y_{i}}, \pi_{i} \not \nrightarrow \Gamma_{y_{i+1}} \mid \operatorname{deg}\left(u_{0}\right) \geq k\right) \leq \varepsilon_{2.1} / 3 .
$$

Let $S_{y_{i}}$ be the number of half-edges and $V_{y_{i}}$ be the number of vertices in $\Gamma_{y_{i}}$, respectively. Then,

$$
V_{y_{i}}=n\left[1-F_{n}\left(y_{i}\right)\right], \quad \text { and } \quad S_{y_{i}} \geq y_{i} n\left[1-F_{n}\left(y_{i}\right)\right],
$$

so that

$$
\mathbb{P}\left(E_{i}\right) \leq\left(1-\frac{S_{y_{i+1}}}{\mathcal{L}_{n}}\right)^{y_{i} / 2} \leq \exp \left\{-\frac{y_{i+1} y_{i}\left[1-F_{n}\left(y_{i+1}\right)\right]}{n \beta}\right\} .
$$

Here $\mathcal{L}_{n} \leq \beta n$ is the total number of half-edges in the graph and the factor $y_{i} / 2$ in the exponent comes from the worst-case scenario in which we connect all the half-edges of $\pi_{i}$ back to $\pi_{i}{ }^{2}$ To show (2.6) we show that, uniformly in $n$, for a proper choice of $y_{i}=y_{i}(k)$,

$$
\sum_{i=0}^{\infty} \exp \left\{-\frac{y_{i+1} y_{i}\left[1-F_{n}\left(y_{i+1}\right)\right]}{n \beta}\right\}=o_{k}(1) .
$$

For this we introduce the shorthand notation for the absolute value of the exponent in (2.9),

$$
g_{i}\left(y_{i}, y_{i+1}\right):=\frac{y_{i+1} y_{i}\left[1-F_{n}\left(y_{i+1}\right)\right]}{n \beta},
$$

where we suppress the dependence of $g_{i}$ on $n$, as we will prove a lower bound on $g_{i}\left(y_{i}, y_{i+1}\right)$ that is uniform in $n$. Using the assumption (2.1), we bound $g_{i}$ as

$$
g_{i}\left(y_{i}, y_{i+1}\right) \geq \tilde{c} y_{i} y_{i+1}^{2-\tau} \exp \left\{-C_{1}\left(\log \left(y_{i+1}\right)\right)^{\gamma}\right\}=\tilde{c} y_{i} y_{i+1}^{2-\tau-C_{1} \log \left(y_{i+1}\right)^{\gamma-1}},
$$

\footnotetext{
1 This statement requires proof. It will follow from the fact that $\pi_{b}$ has degree at least $y_{b}$

2 Here we ignore the possibility that $\pi_{i+1}=\pi_{i}$ for some $i$, as well as the fact that the number of available half-edges decreases while building the path between the different $\pi_{i}$. These effects are indeed negligible, since $V_{y_{i}}$ and $S_{y_{i}}$ are much larger than the number of vertices and half-edges used in the construction of the path.
} 
for some $\tilde{c}>0$. We would like to choose the sequence $\left(y_{i}\right)_{i \geq 1}$ so that (2.9) holds and then we can choose $k=k(\varepsilon)$ in Proposition 2.1 large enough so that (2.9) is at most $\varepsilon / 2$, giving (2.6). We claim that a sequence satisfying these conditions is given recursively by

$$
y_{0}=k, \quad y_{i+1}=y_{i}^{1 /\left(\tau-2+B\left(\log \left(y_{i}\right)\right)^{\gamma-1}\right)},
$$

with $\gamma$ as in (1.1) and $B>0$ to be defined later on. We give upper and lower bounds on $\left(y_{i}\right)_{i \geq 0}$ in the following lemma:

Lemma 2.2 For every $\delta>0$ small enough, there exists $k \in \mathbb{N}$ large enough such that, if $y_{0}=k$, for all $i \geq 1$,

$$
k^{1 /(\tau-2+\delta)^{i}} \leq y_{i} \leq k^{1 /(\tau-2)^{i}} .
$$

Proof The sequence $y_{i}$ is monotone increasing when $k$ is large enough. Hence, if $y_{0}>k_{0}$ for $k_{0}$ sufficiently large, since $\gamma-1<0$, it holds that

$$
\tau-2+B\left(\log y_{i}\right)^{\gamma-1}<\tau-2+B\left(\log y_{0}\right)^{\gamma-1}<1 .
$$

For a choice of $y_{0}$ satisfying the second inequality in (2.14), we define $\delta:=B\left(\log y_{0}\right)^{\gamma-1}$. We now get a lower bound on $y_{i}$ using

$$
y_{i+1} \geq y_{i}^{1 /(\tau-2+\delta)} \geq \cdots \geq y_{0}^{1 /(\tau-2+\delta)^{i}}=k^{1 /(\tau-2+\delta)^{i}},
$$

while an upper bound is obtained by omitting the term $B \log \left(y_{i}\right)^{\gamma-1}$, which is non-negative, in the exponent, so that, recursively,

$$
y_{i+1} \leq y_{i}^{1 /(\tau-2)} \leq \cdots \leq y_{0}^{1 /(\tau-2)^{i}}=k^{1 /(\tau-2)^{i}} .
$$

This concludes the proof of Lemma 2.2.

We now prove that (2.9) holds for $\left(y_{i}\right)_{i \geq 0}$ as in (2.12):

Lemma 2.3 For an appropriate choice of $B$ in (2.12), with $y_{0}=k$ and uniformly in $n,(2.9)$ holds, and hence (2.6) holds for all $k$ sufficiently large.

Proof Recall $g_{i}\left(y_{i}, y_{i+1}\right)$ from (2.10). We use the lower bound on $g_{i}\left(y_{i}, y_{i+1}\right)$ in (2.11), where we replace $y_{i+1}$ in the base with the recursion in (2.12). Then we obtain

$$
\begin{aligned}
& g_{i}\left(y_{i}, y_{i+1}\right) \geq \tilde{c} y_{i}^{1-\left(\tau-2+C_{1}\left(\log \left(y_{i+1}\right)\right)^{\gamma-1}\right) /\left(\tau-2+B\left(\log \left(y_{i}\right)\right)^{\gamma-1}\right)} \\
& =\tilde{c} y_{i}^{\left(B\left(\log y_{i}\right)^{\gamma-1}-C_{1}\left(\log y_{i+1}\right)^{\gamma-1}\right) /\left(\tau-2+B\left(\log y_{i}\right)^{\gamma-1}\right)} .
\end{aligned}
$$

We investigate the numerator in the exponent on the rhs. Since $y_{i}$ is monotone increasing, for the choice of $B>2 C_{1}$ we get that

$$
B\left(\log y_{i}\right)^{\gamma-1}-C_{1}\left(\log y_{i+1}\right)^{\gamma-1} \geq 2 C_{1}\left(\log y_{i}\right)^{\gamma-1}-C_{1}\left(\log y_{i}\right)^{\gamma-1} \geq C_{1}\left(\log y_{i}\right)^{\gamma-1} .
$$

Using this bound in the numerator, and that $\gamma-1 \leq 0$ implies that for $k$ large enough, uniformly in $i, \tau-2+B \log \left(y_{i}\right)^{\gamma-1} \leq \tau-2+\delta^{\prime}<1$ followed by the lower bound on $y_{i}$ in Lemma 2.2, we obtain for some constant $C$,

$$
g_{i}\left(y_{i}, y_{i+1}\right) \geq \tilde{c} \exp \left\{C\left(\log y_{i}\right)^{\gamma}\right\} \geq \tilde{c} \exp \left\{C(\log k)^{\gamma}\left(\frac{1}{\tau-2+\delta}\right)^{i \gamma}\right\} .
$$

Note that $\tau-2+\delta<1$, so that $\exp \left\{-g_{i}\left(y_{i}, y_{i+1}\right)\right\}$ is dominated by a geometric series, hence it is summable in $i$ (uniformly in $n$ ), and is a constant times its first term. This constant 
is less than 2 whenever $k$ is large enough so that $\exp \left\{-g_{0}\left(y_{0}, y_{1}\right)\right\} \leq 1 / 2$. As a result, since $\gamma>0$ and $y_{0}=k$,

$$
\sum_{i=0}^{\infty} \exp \left\{-g_{i}\left(y_{i}, y_{i+1}\right)\right\} \leq 2 \exp \left\{-\exp \left\{C(\log k)^{\gamma}\right\}\right\} \rightarrow 0,
$$

as $k \rightarrow \infty$, establishing the statement of Lemma 2.3 as well as (2.9).

We want to give an upper bound on $b$, i.e., the index $i$ for which $\pi_{i}$ is connected to $\Lambda_{\eta}, \eta>1 / 2$, the set of vertices of high degree. For this, we refine the lower bound in Lemma 2.2 in the next lemma:

Lemma 2.4 There exists $\delta \in(0,1)$ such that, for $k$ sufficiently large,

$$
y_{i} \geq\left(k^{1-\delta}\right)^{(\tau-2)^{-i}} .
$$

Proof Let us write $y_{i+1}=k^{a_{i+1}}$, so that by (2.12),

$$
a_{i+1}=\frac{a_{i}}{\tau-2+B\left(\log y_{i}\right)^{\gamma-1}}=\cdots=\prod_{j=0}^{i}\left(\tau-2+B\left(\log y_{j}\right)^{\gamma-1}\right)^{-1} .
$$

Using the lower bound on $y_{i}$ in Lemma 2.2 we get

$$
a_{i+1} \geq(\tau-2)^{-(i+1)} \prod_{j=0}^{i}\left(1+(\tau-2+\delta)^{-j(\gamma-1)} \frac{B(\log k)^{\gamma-1}}{\tau-2}\right)^{-1} .
$$

The convergence of the product on the rhs of (2.22) for any fixed $k$ as $i \rightarrow \infty$ is equivalent to the convergence of the series

$$
(B \log k)^{1-\gamma} \sum_{j=0}^{i}(\tau-2+\delta)^{j(1-\gamma)},
$$

and this series converges as $i \rightarrow \infty$ because $\tau-2+\delta<1$ for $\delta>0$ sufficiently small. So, let us write

$$
M_{k}^{-1}:=\lim _{i \rightarrow \infty} \prod_{j=0}^{i}\left(1+(\tau-2+\delta)^{j(1-\gamma)} \frac{B(\log k)^{\gamma-1}}{\tau-2}\right)
$$

and since the partial products on the rhs of (2.24) increase to the limit $M_{k}^{-1}$, combining this with (2.22) yields the lower bound

$$
a_{i+1} \geq \frac{1}{(\tau-2)^{i+1}} M_{k}
$$

Further observe that due to $\gamma<1, M_{k}=1+o_{k}(1)$, therefore, using the form $y_{i}=k^{a_{i}}$ again,

$$
y_{i} \geq\left(k^{M_{k}}\right)^{1 /(\tau-2)^{i}} \geq\left(k^{1-\delta}\right)^{1 /(\tau-2)^{i}}
$$

by taking $k$ so large that $M_{k} \geq 1-\delta$. 
Now we have all the preliminaries to complete the proof of Proposition 2.1:

Proof of Proposition 2.1 By condition (2.1) in Proposition 2.1, there are some vertices in the graph of degree $n^{\eta}$, for $\eta>1 / 2$. As a consequence of Lemma 2.4, the number of layers needed to reach the highest-degree vertices in $\Lambda_{\eta}$ has $b$ as an upper bound, ${ }^{3}$ where $b-1$ is the solution of

$$
\left(k^{1-\delta}\right)^{1 /(\tau-2)^{i}}=n^{\eta},
$$

that is, by elementary calculations,

$$
b \leq \frac{\log \left(\log \left(n^{\eta}\right) / \log y_{0}^{1-\delta}\right)}{\log \left(\frac{1}{\tau-2}\right)}+1=\frac{\log \log n+\log \eta-\log \left(\log \left(y_{0}^{1-\delta}\right)\right)}{|\log (\tau-2)|}+1 .
$$

Then, for $y_{0}=k$ sufficiently large,

$$
b \leq \frac{\log \log n}{|\log (\tau-2)|}=b(n) .
$$

By Lemma 2.3, for all $k \geq k_{0}(\varepsilon)$, (2.6) holds. As a consequence of (2.6), w.h.p., we can connect the vertex $u$ and $v$ with degree $k$, to the set $\Lambda_{\eta}$ with probability $1-2 \varepsilon_{2.1} / 2$ in at most $2 b$ steps. As a consequence of [6, Lemma 5], $\Lambda_{\eta}$ is a complete graph w.h.p., i.e., for all $n \geq n\left(\varepsilon_{2.1}\right)$, the probability that the edge between $\pi_{b}(u)$ and $\pi_{b}(v)$ is not present is at most $\varepsilon_{2.1} / 3$. Hence, we can connect $u_{k}$ and $v_{k}$ in $2 b+1$ steps with probability at least $1-\varepsilon_{2.1}$. That is,

$$
\mathbb{P}\left(\mathcal{E}_{n}^{c} \mid d_{u} \geq k, d_{v} \geq k\right)<\varepsilon_{2.1}
$$

This ends the proof of Proposition 2.1(a). The proof of Proposition 2.1(b) is identical. We mention that the probability that $u, v$ are not connected is estimated along the construction of the path $\pi$, namely, $\left\{\mathcal{D}_{n}(u, v)=\infty\right\} \subset\left\{\exists \pi_{i}: \pi_{i} \nrightarrow \Gamma_{i+1}\right\}$, and hence it is swallowed in the terms in (2.6).

\section{Degree Percolation}

In this section our goal is to define degree-dependent percolation on the configuration model and relate it to an appropriately chosen configuration model. Degree-dependent percolation means that we keep each edge with a probability that depends on the degree of the two vertices the edge is adjacent to. In what follows, we explain two different ways to do this, and show that they are in fact equivalent. Let

$$
p(d): \mathbb{N} \longrightarrow[0,1]
$$

be a monotone decreasing function of $d$. Later $p(d)$ will equal the probability of keeping a half-edge that is attached to a vertex with degree $d$. We now define two different degree-dependent percolation methods given a function $p(d)$, one where we remove edges independently and one where we remove half-edges independently. In each case, the removal probabilities depend on the degree(s) of the vertices involved in the (half-)edge or edge. We start by defining the edge degree-percolation model:

3 This is an upper bound since the true number is an integer, while the solution of (2.27) is not necessarily an integer 
Definition 3.1 (Edge degree-percolation) Consider a configuration model $\mathrm{CM}_{n}(\boldsymbol{d})$ where half-edges are already paired into edges. Keep any edge between vertices $u, v$ in the graph with probability $p\left(d_{u}\right) p\left(d_{v}\right)$, independently for different edges, where $d_{u}$ and $d_{v}$ are the degrees of $u$ and $v$. We denote the graph spanned by kept edges by $\widetilde{\mathrm{CM}}_{n}^{p(d)}(\boldsymbol{d})$.

We let $v(s)$ be the vertex that the half-edge $s$ is attached to and $d_{v(s)}$ its degree. Then half-edge degree-percolation is defined as follows:

Definition 3.2 (Half-edge degree-percolation) Fix a degree sequence $\boldsymbol{d}=\left(d_{1}, \ldots, d_{n}\right)$. We do the following operations:

1. We keep the half-edge $s$ with probability $p\left(d_{v(s)}\right)$, independently across half-edges. Let $d_{v}^{r}$ denote the random number of kept half-edges attached to vertex $v$, and let $\boldsymbol{d}^{r}:=$ $\left(d_{1}^{r}, \ldots, d_{n}^{r}\right)$.

2. If we do not keep the half-edge $s$, then we create a new vertex of degree 1 with one half-edge that corresponds to the deleted half-edge, carrying the same half-edge label $s$. We call the newly created vertex and half-edge artificial. We denote the total number of artificial vertices by $A$ and let $\mathbf{1}(A)$ denote a vector of $A$ many ones.

3. We pair all the half-edges uniformly at random (including the artificial ones), obtaining the configuration model $\mathrm{CM}_{n+A}\left(\boldsymbol{d}^{r}, \mathbf{1}(A)\right)$ with random number $n+A$ many vertices and random degree sequence $\left(\boldsymbol{d}^{r}, \mathbf{1}(A)\right)$.

4. We take the induced subgraph on the first $n$ (original) vertices (i.e., remove the edges that contain at least one artificial half-edge), with isolated vertices removed. We denote the resulting graph by $\mathrm{CM}_{n}^{p(d)}(\boldsymbol{d})$.

Note that the total number of half-edges in $\mathrm{CM}_{n+A}\left(\boldsymbol{d}^{r}, \mathbf{1}(A)\right)$ is again $\mathcal{L}_{n}$ as it was for $\mathrm{CM}_{n}(\boldsymbol{d})$. The final graph $\mathrm{CM}_{n}^{p(d)}(\boldsymbol{d})$ is a subgraph of this configuration model. We will apply Proposition 2.1 to the configuration model $\mathrm{CM}_{n+A}\left(\boldsymbol{d}^{r}, \mathbf{1}(A)\right)$, so it is convenient that $\mathrm{CM}_{n+A}\left(\boldsymbol{d}^{r}, \mathbf{1}(A)\right)$ both encodes the half-edge degree percolation on $\mathrm{CM}_{n}(\boldsymbol{d})$, as well as equals a configuration model itself.

We adapt an argument by Janson [12], who studies various types of percolation on random graphs, including degree-dependent site percolation, to our edge degree-percolation.

Lemma 3.3 Fix a degree sequence $d=\left(d_{1}, \ldots, d_{n}\right)$. Then, $\mathrm{CM}_{n}^{p(d)}(\boldsymbol{d})$ and $\widetilde{\mathrm{CM}}_{n}^{p(d)}$ (d) have the same law. Moreover, ${\widetilde{\mathrm{CM}_{n}^{p}}}^{p(d)}(\boldsymbol{d})$ can be coupled to $\mathrm{CM}_{n+A}\left(\boldsymbol{d}^{r}, \mathbf{1}(A)\right)$ in Definition 3.2

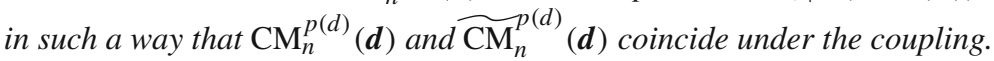

Proof of Lemma 3.3 Given the original list of degrees $\boldsymbol{d}=\left(d_{1}, \ldots, d_{n}\right)$ in $\mathrm{CM}_{n}(\boldsymbol{d})$ that we think of now as labelled half-edges attached to vertices in $[n]$, the outcome ${\widetilde{\mathrm{CM}_{n}^{p(d)}}}_{(\boldsymbol{d})}$ of the edge degree-percolation as well as the outcome $\mathrm{CM}_{n}^{p(d)}(\boldsymbol{d})$ of the half-edge degreepercolation can be described as a sub-matching $\mathcal{M}_{\text {kept }}$ of $\boldsymbol{d}$ that contains the kept and matched half-edges. Hence, for the distributional identity between $\mathrm{CM}_{n}^{p(d)}(\boldsymbol{d})$ and $\widetilde{\mathrm{CM}}_{n}^{p(d)}(\boldsymbol{d})$, we need to show that for every given sub-matching $m^{\star}$ of the labelled half-edges $\boldsymbol{d}$,

$$
\mathbb{P}\left(\mathrm{CM}_{n}^{p(d)}(\boldsymbol{d})=m^{\star}\right)=\mathbb{P}\left(\widetilde{C M}_{n}^{p(d)}(\boldsymbol{d})=m^{\star}\right) .
$$

We start to investigate the rhs. Recall that to obtain ${\widetilde{\mathrm{CM}_{n}}}^{p(d)}(\boldsymbol{d})$, first we take a matching $m$ of $\boldsymbol{d}$ and then for each pair of half-edges $(s, t) \in m$, we decide to keep or delete the edge 
independently with probability $p\left(d_{v(s)}\right) p\left(d_{v(t)}\right)$ and $1-p\left(d_{v(s)}\right) p\left(d_{v(t)}\right)$, respectively, where recall that $v(q)$ denotes the vertex to which the half-edge $q$ is attached to.

Let us denote all the matchings of the labels $\boldsymbol{d}$ that contain the sub-matching $m^{\star}$ by $\mathcal{M}_{m^{\star}}$, and the set of all matchings by $\mathcal{M}$. Recall that every matching $m$ in the configuration model has probability $1 /\left(\mathcal{L}_{n}-1\right)$ !!, where $\mathcal{L}_{n}=\sum_{i=1}^{n} d_{i}$, and that given the matched edges in $m$, we keep every edge $(s, t)$ with probability $p\left(d_{v(s)}\right) p\left(d_{v(t)}\right)$ independently of each other. As a result, we compute the probability to obtain the sub-matching $m^{\star}$ as the outcome of the edge degree-percolation by summing over all possible matchings $m \in \mathcal{M}_{m^{\star}}$ and keeping an edge in $m$ precisely when it is also part of $m^{\star}$ :

$$
\begin{aligned}
\mathbb{P}_{\left.\mathrm{CM}_{n}^{p(d)}(\boldsymbol{d})=m^{\star}\right)=} & \frac{1}{\left(\mathcal{L}_{n}-1\right) ! !} \sum_{m \in \mathcal{M}_{m^{\star}}} \prod_{\left(s^{\prime}, t^{\prime}\right) \in m^{\star}} p\left(d_{v\left(s^{\prime}\right)}\right) p\left(d_{v\left(t^{\prime}\right)}\right) \\
& \times \prod_{(s, t) \in m \backslash m^{\star}}\left(1-p\left(d_{v(s)}\right) p\left(d_{v(t)}\right)\right) .
\end{aligned}
$$

Introducing the indicator $\mathbf{1}_{\{q \mathrm{kept}\}}=1-\mathbf{1}_{\{q \text { not kept }\}}$ for half-edge $q$, we can interpret the factors in the first product as $\mathbb{E}\left[\mathbf{1}_{\{s \text { kept }\}} \mathbf{1}_{\{t \text { kept }\}}\right]$, while we can rewrite the factors in the last product by using the identity

$$
\begin{aligned}
1 & -p\left(d_{v(s)}\right) p\left(d_{v(t)}\right) \\
& =\left(1-p\left(d_{v(s)}\right) p\left(d_{v(t)}\right)+p\left(d_{v(s)}\right)\left(1-p\left(d_{v(t)}\right)\right)+\left(1-p\left(d_{v(s)}\right)\left(1-p\left(d_{v(t)}\right)\right.\right.\right. \\
& =\mathbb{E}\left[\mathbf{1}_{\{s \text { not kept }\}} \mathbf{1}_{\{t \text { not kept }\}}+\mathbf{1}_{\{s \text { kept }\}} \mathbf{1}_{\{t \text { not kept }\}}+\mathbf{1}_{\{s \text { not kept }\}} \mathbf{1}_{\{t \text { not kept }\}}\right] .
\end{aligned}
$$

For each half-edge $s$, let $U_{s}$ a uniform r.v. in [0,1], independent for different $s$. Then one can actually realise the above indicators by setting $\mathbf{1}_{\{s \text { kept }\}}:=\mathbf{1}_{\left\{U_{s} \leq p\left(d_{v(s)}\right)\right\}}$. Note that the events $\mathbf{1}_{\{s \mathrm{kept}\}}$ and $\mathbf{1}_{\{s \text { not kept\}}\}}$ can be also interpreted as the half-edge $s$ being additionally labelled artificial and regular, respectively. Note that the set of half-edges additionally labelled artificial uniquely determine this additional labelling. By writing $\mathcal{A}, \mathcal{A}^{c}$ for the set of half-edges that are additionally labelled artificial/regular, one can rewrite (3.3) as

$$
\begin{aligned}
& \left.\mathbb{P}{\widetilde{\left(\mathbf{C M}_{n}^{p}\right.}}^{p(d)}(\boldsymbol{d})=m^{\star}\right) \\
& =\frac{1}{\left(\mathcal{L}_{n}-1\right) ! !} \sum_{m \in \mathcal{M}_{m^{\star}}} \prod_{(s, t) \in m^{\star}} \mathbb{P}\left(s \in \mathcal{A}^{c}, t \in \mathcal{A}^{c}\right) \times \prod_{(s, t) \in m \backslash m^{\star}} \mathbb{P}(\{s \in \mathcal{A}\} \cup\{t \in \mathcal{A}\}) \\
& =\frac{1}{\left(\mathcal{L}_{n}-1\right) ! !} \sum_{m \in \mathcal{M}_{m^{\star}}} \mathbb{P}\left(\bigcap_{(s, t) \in m^{\star}}\left\{s \in \mathcal{A}^{c}, t \in \mathcal{A}^{c}\right\} \bigcap_{(s, t) \in \subset m \backslash m^{\star}}\{s \in \mathcal{A}\} \cup\{t \in \mathcal{A}\}\right) .
\end{aligned}
$$

Let us thus denote a configuration model, with additional labels 'artificial' on the deterministic set $\mathcal{S}$ and degree sequence $\boldsymbol{d}$ by $\mathrm{CM}_{n}(\boldsymbol{d})(\mathcal{S})$. In other words, $\mathrm{CM}_{n}(\boldsymbol{d})(\mathcal{S})$ can be described by the pair $(m, \mathcal{S})$, where $m$ is a matching of $\boldsymbol{d}$ and $\mathcal{S}$ is the set of half-edges additionally labelled artificial. With $m^{\star}, m$ a sub-matching and a matching of $\boldsymbol{d}$, let us call an additional labelling $\mathcal{S}$ of half-edges proper for $\left(m^{\star}, m\right)$ if it has the property that each pair $(s, t) \in m \backslash m^{\star}$ has at least one half-edge labeled artificial, while all half-edges that form edges that are in $m^{\star}$ are labelled regular. Let us denote the set of proper additional labelings of half-edges by $\mathcal{P}\left(m^{\star}, m\right)$. Note that this set is non-empty if and only if $m \in \mathcal{M}_{m^{\star}}$. Let us further denote by $\mathcal{A}$ a random labelling where each half-edge $s$ gets the regular additional label independently with probability $p\left(d_{v(s)}\right)$, otherwise artificial. With this notation, (3.5) turns into 


$$
\begin{aligned}
\mathbb{P}\left({\widetilde{\mathrm{CM}_{n}^{p(d)}}}^{\left.p(\boldsymbol{d})=m^{\star}\right)}\right. & =\frac{1}{\left(\mathcal{L}_{n}-1\right) ! !} \sum_{m \in \mathcal{M}} \sum_{\mathcal{S} \in \mathcal{P}\left(m^{\star}, m\right)} \mathbb{P}(\mathcal{A}=\mathcal{S}) \\
& =\sum_{m \in \mathcal{M}} \sum_{\mathcal{S} \in \mathcal{P}\left(m^{\star}, m\right)} \mathbb{P}\left(\mathrm{CM}_{n}(\boldsymbol{d})(\mathcal{A})=(m, \mathcal{S})\right),
\end{aligned}
$$

where we have obtained the rhs by moving the prefactor $1 /\left(\mathcal{L}_{n}-1\right)$ !! into the probability sign by using the notion of the additionally labelled configuration model $\mathrm{CM}_{n}(\boldsymbol{d})(\mathcal{S})$ introduced above after (3.5), now with random additional labels $\mathcal{A}$. Note also that the summation goes over all $m \in \mathcal{M}$ since in case $m \notin \mathcal{M}_{m^{\star}}$, the inner sum is empty.

To move towards half-edge degree-percolation, it is left to reverse the two sums in (3.6). For this, let us write $\mathcal{L} a b(\boldsymbol{d})$ for the set of additional labelings of $\boldsymbol{d}$. For a given $\mathcal{S} \in \mathcal{L} a b(\boldsymbol{d})$ and sub-matching $m^{\star}$ of $\boldsymbol{d}$, write $\mathcal{M}\left(m^{\star}, \mathcal{S}\right)$ for the set of matchings that have the property that $\left\{(s, t) \in m: s \in \mathcal{S}^{c}, t \in \mathcal{S}^{c}\right\}=m^{\star}$, i.e., the sub-matching consisting of pairs of half-edges with both regular additional label is $m^{\star}$. In other words, $\mathcal{M}\left(m^{\star}, \mathcal{S}\right)$ contains all those matchings $m$ that extend the sub-matching $m^{\star}$ into a full matching in a way that the edges that are not in $m^{\star}$ contain at least one half-edge that is additionally labelled artificial. Note that for a given additional labelling $\mathcal{S} \in \mathcal{L} a b(\boldsymbol{d})$, the set $\mathcal{M}\left(m^{\star}, \mathcal{S}\right)$ might be empty (e.g., when all the half-edges are labelled regular, or when some half-edge in $m^{\star}$ is labelled artificial). Then, note that $\mathcal{S} \in \mathcal{P}\left(m^{\star}, m\right)$ if and only if $m \in \mathcal{M}\left(m^{\star}, \mathcal{S}\right)$. With the notation $\left.\mathrm{CM}_{n}(\boldsymbol{d})(\mathcal{A})\right|_{1}$ meaning the random matching in $\mathrm{CM}_{n}(\boldsymbol{d})(\mathcal{A})$, (i.e., the first coordinate) the rhs of (3.6) equals

$$
\begin{aligned}
& \sum_{\mathcal{S} \in \mathcal{L} a b(\boldsymbol{d})} \sum_{m \in \mathcal{M}\left(m^{\star}, \mathcal{S}\right)} \mathbb{P}\left(\mathrm{CM}_{n}(\boldsymbol{d})(\mathcal{A})=(m, \mathcal{S})\right) \\
& =\sum_{\mathcal{S} \in \mathcal{L} a b(\boldsymbol{d})} \mathbb{P}\left(\mathcal{A}=\mathcal{S},\left.\mathrm{CM}_{n}(\boldsymbol{d})(\mathcal{A})\right|_{1} \in \mathcal{M}\left(m^{\star}, \mathcal{S}\right)\right) \\
& =\mathbb{P}\left(\left.\mathrm{CM}_{n}(\boldsymbol{d})(\mathcal{A})\right|_{1} \in \mathcal{M}\left(m^{\star}, \mathcal{A}\right)\right)
\end{aligned}
$$

where we brought in the disjoint sum $\sum_{\mathcal{S} \in \mathcal{L} a b(\boldsymbol{d})}$ as a disjoint union to obtain the second line, where now the additional labelling $\mathcal{A}$ is random and distributed as explained before (3.6). Note that one can express the event $\operatorname{CM}_{n}(\boldsymbol{d})(\mathcal{A}) \in \mathcal{M}\left(m^{\star}, \mathcal{A}\right)$ in words as $\left\{\right.$ in $\operatorname{CM}_{n}(\boldsymbol{d})(\mathcal{A})$ the sub-matching spanned by regular half-edges equals $\left.m^{\star}\right\}$. This is already similar to the event defined in Definition 3.2.

It is only left to connect $\mathrm{CM}_{n}(\boldsymbol{d})(\mathcal{A})$ to $\mathrm{CM}_{n+A}\left(\boldsymbol{d}^{r}, \mathbf{1}(A)\right)$. Indeed, in the procedure creating $\mathrm{CM}_{n+A}\left(\boldsymbol{d}^{r}, \mathbf{1}(A)\right)$ in point 3. of Definition 3.2, the half-edges attached to degreeone artificial vertices keep carrying the original label $s$ of the half-edge. Both for the event $\left\{\left.\mathrm{CM}_{n}(\boldsymbol{d})(\mathcal{A})\right|_{1} \in \mathcal{M}\left(m^{\star}, \mathcal{A}\right)\right\}$, as well as for the event $\{$ the graph spanned by regular halfedges equals $m^{\star}$ in $\left.\mathrm{CM}_{n+A}\left(\boldsymbol{d}^{r}, \mathbf{1}(A)\right)\right\}$, artificial half-edges and isolated vertices will be removed when taking the subgraph $\mathrm{CM}_{n}^{p(d)}(\boldsymbol{d})$, so it makes no difference whether we consider the half-edge $s$ additionally labelled 'artificial' or actually we first remove it and attach it to a degree-one vertex. Further, by exchangeability, $\left.\mathrm{CM}_{n+A}\left(\boldsymbol{d}^{r}, \mathbf{1}(A)\right)\right\}$ has the same law when we re-label the artificial half-edges in the vector $\mathbf{1}(A)$. Hence, we arrive at

$$
\begin{aligned}
& \mathbb{P}\left(\widetilde{C M}_{n}^{p(d)}(\boldsymbol{d})=m^{\star}\right)=\mathbb{P}\left(\left.\mathrm{CM}_{n}(\boldsymbol{d})(\mathcal{A})\right|_{1} \in \mathcal{M}\left(m^{\star}, \mathcal{A}\right)\right) \\
& =\mathbb{P}\left(\operatorname{In} \mathrm{CM}_{n+A}\left(\boldsymbol{d}^{r}, \mathbf{1}(A)\right) \text { the sub-matching spanned by regular half-edges equals } m^{\star}\right\} \\
& =\mathbb{P}\left(\mathrm{CM}_{n}^{p(d)}(\boldsymbol{d})=m^{\star}\right)
\end{aligned}
$$


This finishes the proof of (3.2). Note that we could prove this statement for sub-matchings $m^{\star}$ of $\boldsymbol{d}$, but, by exchangeability, one can obtain the same result for graphs $G$ as outcome, where only the vertices are labelled from $[n]$ (and half-edge labels are forgotten), by taking a union over all possible labellings of the two ends of the edges of $G$. In other words, for two labellings $\mathcal{S}, \mathcal{S}^{\prime}$ that leave the same regular degree sequence $\boldsymbol{d}^{r}$, the probability that the subgraph spanned by regular edges equals $G$ is the same. In other words, by exchangeability, one can look at $\mathrm{CM}_{n+A}\left(\boldsymbol{d}^{r}, \mathbf{1}(A)\right)$ with arbitrary half-edge labels on $\mathbf{1}(A)$.

Finally, in our proof, the additional labels 'artificial' and 'regular' correspond to the indicators $\mathbf{1}_{\left\{U_{s} \geq p\left(d_{v(s)}\right)\right\}}$ and $\mathbf{1}_{\left\{U_{s} \leq p\left(d_{v(s)}\right)\right\}}$, determined by i.i.d. uniform half-edge weights, respectively, see (3.4). Since half-edge labels are kept when creating an artificial vertex, one can use the same random variable $U_{s}$ in the two notions of percolation, and then the corresponding indicators 'carry through' $\mathrm{CM}_{n+A}\left(d^{r}, \mathbf{1}(A)\right)$ as well, when the 1's in $\mathbf{1}(A)$ do carry the original half-edge labels $s$. This provides the coupling and finishes the proof.

Remark 3.4 (Paths in $\mathrm{CM}_{n+A}\left(\boldsymbol{d}^{r}, \mathbf{1}(A)\right)$ ) If $\pi$ is a path in $\mathrm{CM}_{n+A}\left(\boldsymbol{d}^{r}, \mathbf{1}(A)\right)$ consisting only of vertices in $[n]$, then it is also part of $\mathrm{CM}_{n}^{p(d)}(\boldsymbol{d})$. Indeed, the induced subgraph on $[n]$ keeps every path like that.

In reverse, if $\pi$ is a path in $\mathrm{CM}_{n+A}\left(\boldsymbol{d}^{r}, \mathbf{1}(A)\right)$ with degrees $d_{v}^{r} \geq 2$, then, $\pi$ must belong to the subgraph $\mathrm{CM}_{n}^{p(d)}(\boldsymbol{d})$ induced by vertices in $[n]$. Indeed, any artificial vertex has degree 1 so it cannot be part of such $\pi$.

By Lemma 3.3, the law of the percolated graph is equal to a subgraph of a configuration model $\mathrm{CM}_{n+A}\left(\boldsymbol{d}^{r}, \mathbf{1}(A)\right)$ with random degrees $\left(\boldsymbol{d}^{r}, \mathbf{1}(A)\right)$ and random number of vertices $n+$ $A$. In the next results, our goal is to determine the empirical degree distribution of $\left(\boldsymbol{d}^{r}, \mathbf{1}(A)\right)$. Recall we start with a degree sequence $\boldsymbol{d}=\left(d_{1}, \ldots, d_{n}\right)$ that satisfies Assumption 1.1. We would like to maintain a similar power-law condition with the same value of $\tau \in(2,3)$. This is of course not possible for an arbitrary choice of $p(d)$, thus we need to restrict the halfedge retention probabilities to satisfy some degree-dependent bounds. The next proposition is about this:

Proposition 3.5 Consider $\mathrm{CM}_{n}(\boldsymbol{d})$, where the degrees satisfy Assumption 1.1 for some $\tau \in$ $(2,3), \mathcal{L}_{n}=\sum_{i \in[n]} d_{i} \leq n \beta$ for some $\beta<\infty$, and assume that $F_{n}(1)=0$. Perform halfedge degree-percolation on this graph with half-edge retention probability $p(d)$ as described in Definition 3.2. Then, if $p(d)$ satisfies

$$
p(d)>b \exp \left\{-a(\log d)^{\gamma}\right\},
$$

with $\gamma<1$, and $a, b>0$, then the empirical degree distribution $F_{n}^{r}$ of $\left(\boldsymbol{d}^{r}, \mathbf{1}(A)\right)$ also obeys a power law with the same value of $\tau \in(2,3)$. More precisely, for $\alpha$ in Assumption 1.1, there exists $c^{\prime}, C^{\prime}$ such that, for all $x \in\left[1, n^{\alpha}\right)$ and w.h.p.,

$$
1-F_{n}^{r}(x) \geq \frac{c^{\prime}}{x^{\tau-1-C^{\prime}(\log x)^{\gamma-1}}} .
$$

Notice that (3.10) is precisely the condition that is necessary to apply Proposition 2.1. Thus, it allows us to construct a path $\pi$ connecting two vertices of degree at least $k$ in the percolated graph $\mathrm{CM}_{n}^{p(d)}(\boldsymbol{d})$ provided that the degrees of vertices used are at least 2 on $\pi$, by Remark 3.4. To prove Proposition 3.5, we will use the following lemma about concentration of binomial random variables: 
Lemma 3.6 (Concentration of binomial random variables) Let $R$ be a binomial random variable. Then

$$
\mathbb{P}(R \notin[\mathbb{E}[R] / 2,2 \mathbb{E}[R]]) \leq 2 \exp \{-\mathbb{E}[R] / 8\} .
$$

Proof See e.g., [17, Theorem 2.19].

Proof of Proposition 3.5 Let $\boldsymbol{d}$ be the degree sequence in $\mathrm{CM}_{n}(\boldsymbol{d}), p(d)$ the half-edge retention probability, and $\boldsymbol{d}^{r}, F_{n}, F_{n}^{r}$ as above. By monotonicity, we may as well assume that $p(d)=$ $b \exp \left\{-a(\log d)^{\gamma}\right\}$ in (3.9), and we will do so from now on (in the entire paper). The upper bound is obvious since (1.1) implies that

$$
\left[1-F_{n}^{r}\right](x) \leq \frac{n}{n+A}\left[1-F_{n}\right](x) \leq\left[1-F_{n}\right](x) \leq C x^{-\tau+1+C(\log x)^{\gamma-1}} .
$$

For the lower bound, we assume that $x \geq 2$, so that we are only considering vertices that are not artificial. For some $y=y(x)$ to be chosen later,

$$
\left[1-F_{n}^{r}\right](x)=\frac{1}{n+A} \sum_{i \in[n]} \mathbb{1}_{\left\{\operatorname{Bin}\left(d_{i}, p\left(d_{i}\right)\right)>x\right\}} \geq \frac{1}{n+\mathcal{L}_{n}} \sum_{i \in[n]: d_{i} \geq y(x)} \mathbb{1}_{\left\{\operatorname{Bin}\left(d_{i}, p\left(d_{i}\right)\right)>x\right\}} .
$$

Thus, $\left(n+\mathcal{L}_{n}\right)\left[1-F_{n}^{r}\right](x)$ is bounded from below by a sum of independent Bernoulli random variables. We use concentration techniques to show that (3.10) holds w.h.p. Denote

$$
V:=\sum_{i \in[n]: d_{i} \geq y(x)} \mathbb{1}_{\left\{\operatorname{Bin}\left(d_{i}, p\left(d_{i}\right)\right)>x\right\}} .
$$

We start by investigating the mean of $V$. Suppose $y$ is such that $y p(y) \geq 2 x$. Then, for any $i$ for which $d_{i}>y$,

$$
\left.\mathbb{P}\left(\operatorname{Bin}\left(d_{i}, p\left(d_{i}\right)\right)>x\right) \geq \min _{z: z \geq y} \mathbb{P}(\operatorname{Bin}(z, p(z))>x)\right)=: q(x) .
$$

Then, using the monotonicity of $d \mapsto d p(d)$, it holds that $z p(z) \geq y p(y) \geq 2 x$, thus we can apply Lemma 3.6 to obtain

$$
\min _{z: z \geq y}\left(\mathbb{P}(\operatorname{Bin}(z, p(z))>x) \geq \min _{z: z \geq y}\left(1-\exp \left\{-\frac{z p(z)}{8}\right\}\right)\right.
$$

Again, monotonicity of $p(d)$ implies $z p(z) \geq y p(y)$, so

$$
\min _{z: z \geq y}\left(1-\exp \left\{-\frac{z p(z)}{8}\right\}\right)=1-\exp \left\{-\frac{y p(y)}{8}\right\} \text {. }
$$

Since there are $n\left[1-F_{n}\right](y)$ vertices with (original) degree at least $y$, combining this with (3.13), we obtain the lower bound

$$
\mathbb{E}[V] \geq n\left[1-F_{n}\right](y(x)) \cdot q(x) \geq n\left(1-\exp \left\{-\frac{y p(y)}{8}\right\}\right) \frac{c}{y^{\tau-1+C(\log y)^{\gamma-1}}}
$$

Let us set now $y(x):=2 x \exp \left\{2 c\left(\log \frac{2}{a} x\right)^{\gamma}\right\} / a$, for $\gamma, a, b$ as in (3.9). Since $p(y)$ satisfies the lower bound given in (3.9),

$$
\begin{aligned}
y(x) p(y(x)) & =2 x \exp \left\{2 c\left(\log \frac{2}{a} x\right)^{\gamma}-c\left(\log \frac{2}{a} x+2 c\left(\log \frac{2}{a} x\right)^{\delta}\right)^{\gamma}\right\} \\
& =2 x \exp \left\{2 c\left(\log \frac{2}{a} x\right)^{\gamma}-c\left(\log \frac{2}{a} x\right)^{\gamma}\left(1+2 c\left(\log \frac{2}{a} x\right)^{\gamma-1}\right)^{\gamma}\right\} .
\end{aligned}
$$


Note that since $\gamma<1$, the factor $\left(1+2 c\left(\log \frac{2}{a} x\right)^{\gamma-1}\right)^{\gamma}$ is less than say $3 / 2$ (but larger than 1 ) if $x$ is large enough, and hence, for large enough $x$, the rhs is at least

$$
y(x) p(y(x)) \geq 2 x \exp \left\{\frac{1}{2} c\left(\log \frac{2}{a} x\right)^{\gamma}\right\} \geq 2 x .
$$

This shows that we can indeed apply Lemma 3.6 above.

Note that, due to the bound $y p(y) \geq 2 x$, the factor $(1-\exp \{-y p(y) / 8\}) \geq 1 / 2$ for large enough $x$ in (3.18). Using this estimate and again that $y=2 x \exp \left\{2 c\left(\log \frac{2}{a} x\right)^{\gamma}\right\} / b$ we obtain from (3.18)

$$
\begin{aligned}
& {\left[1-F_{n}\right](y(x)) \cdot q(x)} \\
& \quad \geq \frac{c}{2} \frac{1}{\left(\frac{2}{a} x\right)^{\tau-1}} \exp \left\{-(\tau-1)\left(2 c\left(\log \frac{2}{a} x\right)^{\gamma}\right)-C\left(\log \left(\frac{2}{a} x\right)+2 c\left(\log \frac{2}{a} x\right)^{\gamma}\right)^{\gamma}\right\} .
\end{aligned}
$$

As before, the second term in the exponent is $C\left(\log \frac{2}{a} x\right)^{\gamma}\left(1+2 c\left(\log \frac{2}{a} x\right)^{\gamma-1}\right)^{\gamma}$, and, since $\gamma<1$, the latter factor is at most $3 / 2$ when $x$ is sufficiently large. Thus

$$
\left[1-F_{n}\right](y(x)) \cdot q(x) \geq \frac{c a^{\tau-1}}{2^{\tau}} \frac{1}{x^{\tau-1}} \exp \left\{-\left(\frac{3}{2} C+(\tau-1)\right)\left(\log \frac{2}{a} x\right)^{\gamma}\right\} .
$$

Finally, again for sufficiently $\operatorname{large} x, \log \frac{2}{a} x=\log x\left(1+\frac{\log (2 / a)}{\log x}\right) \leq 2 \log x$, so we arrive at

$$
\begin{aligned}
& {\left[1-F_{n}\right](y(x)) \cdot q(x) \geq \frac{c a^{\tau-1}}{2^{\tau}} \frac{1}{x^{\tau-1}} \exp \left\{-2^{\gamma}\left(\frac{3}{2} C+(\tau-1)\right)(\log x)^{\gamma}\right\}} \\
& \quad=: \frac{\widetilde{c}}{x^{\tau-1}} \exp \left\{-\widetilde{C}(\log x)^{\gamma}\right\}
\end{aligned}
$$

Combining (3.13), (3.18) and (3.22), we see that $\left(n+\mathcal{L}_{n}\right) \mathbb{E}\left[1-F_{n}^{r}\right](x) / n$ satisfies the lower bound in (1.3) in Assumption 1.1 with exponent $\tau, \gamma \in(0,1), c$ replaced by $\widetilde{c}=c a^{\tau-1} / 2^{\tau}$ and $C$ replaced by $\widetilde{C}=2^{\gamma}\left(\frac{3}{2} C+(\tau-1)\right)$.

We next extend the argument to show that the required lower bound on $\left[1-F_{n}^{r}\right](x)$ holds w.h.p. Again suppose $y$ is such that $y p(y) \geq 2 x$. By (3.15)-(3.18) and the independence of the summands in (3.13), $\left(n+\mathcal{L}_{n}\right)\left[1-F_{n}^{r}\right](x) / n$ is bounded from below by a binomial random variable with parameters $n\left[1-F_{n}\right](y(x))$ and success probability $q(x)$, so that, by Lemma 3.6,

$$
\mathbb{P}\left(\left[1-F_{n}^{r}\right](x) \leq n\left[1-F_{n}\right](y(x)) q(x) /\left(2\left(n+\mathcal{L}_{n}\right)\right)<\exp \left\{-n\left[1-F_{n}\right](y(x)) \cdot q(x) / 8\right\},\right.
$$

where we can use the lower bound on $\left[1-F_{n}\right](y(x)) q(x)$ in (3.22) on the opposite of the exponent on the rhs. Since the lower bound, i.e., the rhs of (3.22), is decreasing in $x$, the rhs of (3.23) is bounded from above, for all $x \leq n^{\alpha}$ by

$$
\begin{aligned}
& \mathbb{P}\left(\left[1-F_{n}^{r}\right](x) \leq n\left[1-F_{n}\right](y(x)) q(x) /\left(2\left(n+\mathcal{L}_{n}\right)\right)\right) \\
& \quad \leq \exp \left\{-n \frac{\widetilde{c}}{n^{\alpha(\tau-1)}} \exp \left\{-\widetilde{C} \log \left(n^{\alpha}\right)^{\gamma}\right\}\right\} .
\end{aligned}
$$

Then, since $\alpha<1 /(\tau-1)$ in Assumption 1.1, the opposite of the exponent on the rhs is at least $n^{c}$ for some constant $c>0$. Thus, using the lower bound on $\left[1-F_{n}\right](y(x)) \cdot q(x)$ from 
(3.22) once more, (now inside the probability sign on the lhs of (3.24)), the assumption that $\mathcal{L}_{n} \leq \beta n$, and a union bound,

$$
\begin{aligned}
& \mathbb{P}\left(\exists x \leq n^{\alpha}:\left[1-F_{n}^{r}\right](x) \leq \frac{\widetilde{c} /(2(1+\beta))}{x^{\tau-1}} \exp \left\{-\widetilde{C}(\log x)^{\gamma}\right\}\right) \\
& \leq \sum_{x \leq n^{\alpha}} \mathbb{P}\left(1-F_{n}^{r}(x) \leq\left[1-F_{n}\right](y(x)) q(x) / 2\right) .
\end{aligned}
$$

By (3.23) and (3.24), each summand is at most $\exp \left\{-n^{c}\right\}$. So, we obtain

$$
\mathbb{P}\left(\exists x \leq n^{\alpha}:\left[1-F_{n}^{r}\right](x) \leq \frac{\widetilde{c} /(2(1+\beta))}{x^{\tau-1}} \exp \left\{-\widetilde{C}(\log x)^{\gamma}\right\}\right)<n^{\alpha} \exp \left\{-n^{c}\right\}=o_{n}(1),
$$

as required. This completes the proof of Proposition 3.5 with exponent $\tau, \gamma \in(0,1), c$ replaced by $\widetilde{c} /(2(1+\beta))=c a^{\tau-1} /\left(2^{\tau+1}(1+\beta)\right)$ and $C$ replaced by $\widetilde{C}=2^{\gamma}\left(\frac{3}{2} C+(\tau-1)\right)$.

We now combine Propositions 2.1 and 3.5 to ensure that we can construct a path in the degree-percolated graph. Let $\mathcal{D}_{n}^{r}$ be the graph-distance in ${\widetilde{\mathrm{CM}_{n}}}^{p(d)}(\boldsymbol{d})$. Then, we have the following bound:

Corollary 3.7 Consider $\mathrm{CM}_{n}(\boldsymbol{d})$ with i.i.d. degrees having distribution satisfying (1.1) and $p(d)$ satisfying (3.9), and the corresponding edge-percolated graph $\widetilde{\mathrm{CM}}_{n}^{p(d)}(\boldsymbol{d})$. Then, Proposition 2.1 remains valid for the percolated graph. Namely, for $\mathcal{E}_{n}$ defined in (2.2), let us write

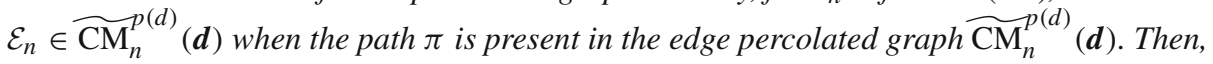
for $\varepsilon_{3.7}>0$, there exists $k=k\left(\varepsilon_{3.7}\right)$ and $n_{0}\left(\varepsilon_{3.7}\right)$ s.t., for all $n \geq n_{0}\left(\varepsilon_{3.7}\right)$, if $u$ and $v$ are two uniformly chosen vertices in $[n]$,

$$
\mathbb{P}\left(\mathcal{E}_{n} \in{\widetilde{\mathrm{CM}_{n}^{p(d)}}}_{(\boldsymbol{d})} \mid d_{u}^{r} \geq k, d_{v}^{r} \geq k\right) \geq 1-\varepsilon_{3.7} .
$$

The same result holds when instead taking $u$ and $v$ uniformly from the set of vertices with degrees $d_{u}^{r}=\ell_{1}$ and $d_{v}^{r}=\ell_{2}$, whenever $\ell_{1}, \ell_{2} \geq k\left(\varepsilon_{3.7}\right)$. As a consequence

$$
\mathbb{P}\left(\mathcal{D}_{n}^{r}-\frac{\log \log n}{\mid \log (\tau-2 \mid}>1 \mid d_{u}^{r} \geq k, d_{v}^{r} \geq k\right) \leq \varepsilon_{3.7} .
$$

Proof By Lemma 3.3, the law of the degree-percolated graph $\widetilde{\mathrm{CM}}_{n}^{p(d)}(\boldsymbol{d})$ can be looked at as subgraph of a new configuration model $\mathrm{CM}_{n+A}\left(\boldsymbol{d}^{r}, \mathbf{1}(A)\right)$ with random degrees $\left(\boldsymbol{d}^{r}, \mathbf{1}(A)\right)$ and random number of vertices $n+A$, and empirical degree distribution $F_{n}^{r}$. Let

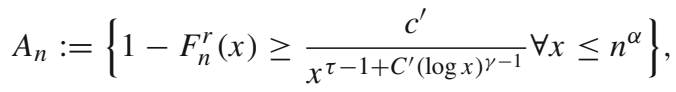

where $\alpha>1 / 2$ is defined in Proposition 3.5. Then by Proposition 3.5, $\mathbb{P}\left(A_{n}\right)=1-o_{n}(1)$.

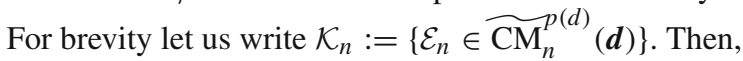

$$
\begin{aligned}
\mathbb{P}\left(\mathcal{K}_{n}^{c} \mid d_{u}^{r} \geq k, d_{v}^{r} \geq k\right)= & \mathbb{P}\left(\mathcal{K}_{n}^{c} \mid d_{u}^{r} \geq k, d_{v}^{r} \geq k, A_{n}\right) \mathbb{P}\left(A_{n}\right) \\
& +\mathbb{P}\left(\mathcal{K}_{n}^{c} \mid d_{u}^{r} \geq k, d_{v}^{r} \geq k, A_{n}^{c}\right) \mathbb{P}\left(A_{n}^{c}\right) .
\end{aligned}
$$

On $A_{n}$, Proposition 2.1 applies to $\mathrm{CM}_{n+A}\left(\boldsymbol{d}^{r}, \mathbf{1}(A)\right)$ as well, the probability of the first term on the rhs is bounded in Proposition 2.1(a), where we also use that the degrees of vertices in 
the path obtained are always at least 2, so that the path is not destroyed by the removal of the artificial vertices and edges (recall Remark 3.4). Further, $\mathbb{P}\left(A_{n}^{c}\right)=o_{n}(1)$ by Proposition 3.5. Thus, with $\varepsilon_{2.1}$,

$$
\mathbb{P}\left(\mathcal{K}_{n} \mid d_{u}^{r} \geq k, d_{v}^{r} \geq k\right) \leq \varepsilon_{2.1}\left(1-o_{n}(1)\right)+o_{n}(1) \leq \varepsilon_{3.7},
$$

for all $n$ sufficiently large, finishing the proof. The same proof works in the setting where $u$ and $v$ are chosen uniformly from the set of vertices with degrees $d_{u}^{r}=\ell_{1}$ and $d_{v}^{r} \geq \ell_{2}$, whenever $\ell_{1}, \ell_{2} \geq k$, now using Proposition 2.1(b).

We finish this section by connecting degree-percolation and the presence of edge-weights.

Claim 3.8 (Edge degree-percolation and excess edge-weights) Consider an edge-weighted $\mathrm{CM}_{n}(\boldsymbol{d})$ where the edges have i.i.d. edge-weights from distribution $1+X_{1}+X_{2}$, with $X_{1}, X_{2}$ i.i.d. random variables, and a non-decreasing function $\left(\xi_{d}\right)_{d \geq 1}$. Then, the following procedure yields an edge degree-percolation model as defined in Definition 3.1:

1. For each edge $e=(z, w)$ with $z, w$ two half-edges, with $z \leq w$ and weight $1+X_{1}^{(e)}+X_{2}^{(e)}$, 'allocate' $X_{1}^{(e)}$ to $z$ and $X_{2}^{(e)}$ to $w$.

2. Keep the edge precisely when the event $\left\{X_{1}^{(e)} \leq \xi_{d_{v(z)}}\right\} \cap\left\{X_{2}^{(e)} \leq \xi_{d_{v(w)}}\right\}$ occurs.

Proof Since the excess edge-weights are all i.i.d., every edge is kept independently. Given $\left(\xi_{d}\right)_{d \geq 1}$, we set $p(d):=\mathbb{P}\left(X \leq \xi_{d}\right)$. The probability of keeping a present edge $(z, w)$ is

$$
\mathbb{P}\left(X_{1} \leq \xi_{d_{v(z)}}, X_{2} \leq \xi_{d_{v(w)}}\right)=\mathbb{P}\left(X_{1} \leq \xi_{d_{v(z)}}\right) \mathbb{P}\left(X_{2} \leq \xi_{d_{v(w)}}\right)=p\left(d_{v(z)}\right) p\left(d_{v(w)}\right),
$$

since $X_{1}, X_{2}$ are independent.

Claim 3.9 (Half-edge degree-percolation and half-edge-weights) Consider a configuration model $\mathrm{CM}_{n}(\boldsymbol{d})$, a random variable $X$, and a non-decreasing function $\left(\xi_{d}\right)_{d \geq 1}$. Then, the following procedure yields a half-edge degree-percolation model as defined in Definition 3.2:

For each half-edge $s$, allocate a random variable $X_{s}$, a copy of $X$, independent for different s. Keep the half-edge s precisely when $\left\{X_{s} \leq \xi_{d_{v(s)}}\right\}$, and then follow the operations in Definition 3.2.

Proof Since the half-edge weights are all i.i.d., every half-edge is kept independently. Given $\left(\xi_{d}\right)_{d \geq 1}$, we set $p(d):=\mathbb{P}\left(X \leq \xi_{d}\right)$. Note that $\mathbb{P}(s$ is kept $)=\mathbb{P}\left(X_{s} \leq \xi_{d_{v(s)}}\right)=p\left(d_{v(s)}\right)$.

Note that one can represent the weight of a (kept or non-kept) edge in the half-edge degreepercolation as the sum of the weights of the two half-edges involved. Hence, combining Claims 3.8, 3.9 with Lemma 3.3 yields the following corollary:

Corollary 3.10 Consider an edge-weighted $\mathrm{CM}_{n}(\boldsymbol{d})$ where the edges have i.i.d. edge-weights from distribution $1+X_{1}+X_{2}$, with $X_{1}, X_{2}$ i.i.d. random variables, and a non-decreasing function $\left(\xi_{d}\right)_{d \geq 1}$. Let $p(d):=F_{X}\left(\xi_{d}\right)$, and realise the edge degree-percolation and halfedge degree percolation (Definitions 3.1, 3.2) on $\mathrm{CM}_{n}(\boldsymbol{d})$ as in Claims 3.8, 3.9. Then, there is a coupling between the two procedures so that the resulting $\mathrm{CM}_{n}^{p(d)}(\boldsymbol{d})$ and $\widetilde{\mathrm{CM}}_{n}^{p(d)}(\boldsymbol{d})$ coincide.

The definition in Sect. 1.3 of the edge-weighted configuration model says that we allocate the edge-weights after the pairing of the half-edges. Hence, one would like to construct paths in $\mathrm{CM}_{n}(\boldsymbol{d})$ that have short excess edge-weight by percolating the already constructed and edgeweighted graph, i.e., a path in ${\widetilde{\mathrm{CM}_{n}}}^{(d)}(\boldsymbol{d})$, an object that is hard to work with. Corollary 3.10 
ensures that we can work with $\mathrm{CM}_{n+A}\left(\boldsymbol{d}^{r}, \mathbf{1}(A)\right)$ instead. The main advantage to working with $\mathrm{CM}_{n+A}\left(\boldsymbol{d}^{r}, \mathbf{1}(A)\right)$ compared to $\mathrm{CM}_{n}^{p(d)}(\boldsymbol{d})$ or ${\widetilde{\mathrm{CM}_{n}^{p(d)}}}_{(\boldsymbol{d})}$, is that it is a configuration model itself.

\section{Tightness of the Weight}

Our goal is to prove Theorem 1.3 in this section. We first connect the uniformly chosen vertex $u$ to a vertex $u_{k}$ with $d_{u_{k}}^{r} \geq k$ in $\mathrm{CM}_{n+A}\left(\boldsymbol{d}^{r}, \mathbf{1}(A)\right)$ and give a bound on the excess weight of this path. We let

$$
\partial \mathcal{B}_{m}(u):=\left\{w \in \mathrm{CM}_{n}(\boldsymbol{d}) \text { s.t. } \mathcal{D}_{n}(u, w)=m\right\},
$$

and define the vertices $V_{m}(u)$ and $V_{m}^{p(d)}(u)$ by

$$
\operatorname{deg}\left(V_{m}(u)\right):=\max _{w \in \partial \mathcal{B}_{m}(u)} d_{w}, \quad \operatorname{deg}\left(V_{m}^{p(d)}(u)\right):=\max _{w \in \partial \mathcal{B}_{m}(u)} d_{w}^{r},
$$

(breaking ties in an arbitrary way, if necessary), where we recall that $d_{w}^{r}$ equals the regular degree of $w$ in $\mathrm{CM}_{n+A}\left(\boldsymbol{d}^{r}, \mathbf{1}(A)\right)$. The following holds:

Lemma 4.1 Assume that $p(d)$ satisfies (3.9) and that $d \mapsto d p(d)$ is monotone increasing. Then, for all $\varepsilon_{4.1}>0$ and for all $k>0$, there exists a $k\left(\varepsilon_{4.1}\right)$ and constant $m:=m\left(k, \varepsilon_{4.1}\right)$ such that

$$
\mathbb{P}\left(\operatorname{deg}\left(V_{m}^{p(d)}(u)\right)<k\right)<\varepsilon_{4.1} .
$$

Proof The proof consists of two steps. In the first step we show that when a vertex has sufficiently high degree before percolation, say $K(k, \varepsilon)$, then after the degree-dependent percolation its regular degree is at least $k$ with probability at least $\varepsilon / 2$. In the second step we determine the graph distance $m$, that we can find a vertex with degree at least $K(k, \varepsilon)$ at graph distance $m$ from the uniformly chosen vertex $u$. Recall Definition 3.2. Since half-edges are kept independently, for any vertex $w \in[n], d_{w}^{r} \stackrel{d}{=} \operatorname{Bin}\left(d_{w}, p\left(d_{w}\right)\right)$.

For any $z>2$, define

$$
L(k, z):=\inf _{d>0}\{d p(d) \geq z k\}
$$

which is well-defined since $d \mapsto d p(d)$ is monotone increasing in $d$. Then, a Chernoff bound as in [15, Corollary 13.3], with $\beta=1-1 / z \geq 1 / 2$ and $\mu=z k$ yields that

$$
\mathbb{P}(\operatorname{Bin}(L(z, k), p(L(z, k)))<k) \leq \mathrm{e}^{-\beta^{2} \mu / 2} \leq \exp \left\{-(1-1 / z)^{2} z k / 2\right\} \leq \exp \{-z k / 8\} .
$$

Thus, for any $\varepsilon_{4.1}>0$ and any $k>0$, choose first $z:=z\left(\varepsilon_{4} .1\right)$ large enough such that $\exp \{-z k / 8\}$ is at most $\varepsilon_{4.1} / 2$, and that gives a $K\left(k, \varepsilon_{4.1}\right):=L\left(k, z\left(\varepsilon_{4.1}\right)\right)$ according to (4.4). This means that with probability at least $\varepsilon_{4.1} / 2$, a vertex with (original) degree at least $K\left(k, \varepsilon_{4.1}\right)$ has regular-degree $d^{r}$ at least $k$.

We now show that we can connect a uniformly chosen vertex $u$ to a vertex with degree at least $K\left(k, \varepsilon_{4} .1\right)$ in a bounded number of steps and probability tending to 1 within $\mathrm{CM}_{n}(\boldsymbol{d})$, i.e., in the original graph. By the coupling to i.i.d. random variables discussed in the proof of Proposition 1.8, in particular, by (1.19),

$$
\left|\partial \mathcal{B}_{m}\right| \stackrel{\mathbb{P}}{\longrightarrow} \infty
$$


and the sequence $\left\{\left(d_{v_{i}}-1\right)_{v_{i} \in \partial \mathcal{B}_{m}}\right\}$ can be coupled to $\left|\partial \mathcal{B}_{m}\right|$ many i.i.d. random variables with a power-law distribution described in (1.4). Then, by (1.18)

$\mathbb{P}\left(\operatorname{deg}\left(V_{m}(u)\right) \leq K\left(k, \varepsilon_{4.1}\right)\right) \leq\left(1-\frac{C^{\star}}{K\left(k, \varepsilon_{4.1}\right)^{\tau-2}} \exp \left\{-C^{\star} \log \left(K\left(k, \varepsilon_{4.1}\right)\right)^{\gamma}\right\}\right)^{\left|\partial \mathcal{B}_{m}\right|}$,

with $\operatorname{deg}\left(V_{m}(u)\right)$ defined in (4.2) and for some positive constant $C^{\star}$. We conclude that, for all $\varepsilon_{4.1}>0$, there exists $m\left(k, \varepsilon_{4.1}\right)$ such that $\mathbb{P}\left(\operatorname{deg}\left(V_{m\left(k, \varepsilon_{4.1}\right.} \leq K\left(k, \varepsilon_{4.1}\right)\right)<\varepsilon_{4.1}\right.$. Finally, let

$$
\begin{aligned}
& \left.E_{1}:=\left\{\operatorname{deg}\left(V_{m\left(k, \varepsilon_{4} .1\right.}(u)\right)<K\left(k, \varepsilon_{4.1}\right)\right)\right\} \\
& \left.E_{2}:=\left\{\operatorname{deg}\left(V_{m\left(k, \varepsilon_{4.1}\right)}(u)\right)>K\left(k, \varepsilon_{4.1}\right)\right), d_{\left.V_{m(k, \varepsilon} 4_{.1}\right)^{(u)}}^{r}<k,\right\}
\end{aligned}
$$

Then, $\mathbb{P}\left(E_{1}^{c}\right)$ is estimated below (4.7) while $\mathbb{P}\left(E_{2}^{c}\right)$ below (4.5), so, by noticing that $\operatorname{deg}\left(V_{m}^{p(d)}(u)\right) \geq d_{\left.V_{m(k, \varepsilon} 4_{.1}\right)^{(u)}}^{r}$ by definition and a union bound,

$$
\mathbb{P}\left(\operatorname{deg}\left(V_{m}^{p(d)}(u)\right)<k\right) \leq \mathbb{P}\left(E_{1}^{c}\right)+\mathbb{P}\left(E_{2}^{c}\right) \leq \varepsilon_{4.1} / 2+\varepsilon_{4.1} / 2 .
$$

This completes the proof of the lemma.

We are now ready to prove Theorem 1.3 by constructing a path with tight excess edgeweight.

Proof of Theorem 1.3 Fix $k$ such that $k>k\left(\varepsilon_{2.1}\right)$, where $k\left(\varepsilon_{2.1}\right)$ is defined in Proposition 2.1. Here $k$ will be determined later on in the proof. Taking $\varepsilon_{4.1}=\varepsilon$ in Lemma 4.1, for any fixed $\varepsilon_{4}$.1 with probability at least $1-\varepsilon_{4}$, , there exists a path in $\operatorname{CM}_{n}(\boldsymbol{d})$ that connects $u$ with a vertex $u_{k}$ with $d_{u_{k}}^{r} \geq k$ in at most $m:=m\left(k, \varepsilon_{4.1}\right)$ steps. We call this path $\kappa=\left(\kappa_{i}\right)_{i \leq k}$, and set $u_{k}:=\kappa_{k}$. We do the same from $v$ giving us a path $\kappa^{\prime}$ and vertex $v_{k}$. Since the choice of these paths did not depend on the excess edge-weights, only on the degrees of vertices, the excess weight over the path $\kappa_{k}$ is given by the sum of at most $m$ i.i.d. random variables with distribution $F_{X}$. Therefore for all $\varepsilon_{(4.10)}>0$ there exists $r=r\left(\varepsilon_{(4.10)}\right)$ such that

$$
\mathbb{P}\left(W_{n}\left(u, u_{k}\right)+W_{n}\left(v, v_{k}\right)>r\right)<\varepsilon_{(4.10)} .
$$

Next we connect $u_{k}, v_{k}$ using edge degree-percolation. We aim to prove an upper bound on the weight of the path that connects $u_{k}$ and $v_{k}$ in the percolated graph $\widetilde{\mathrm{CM}}_{n}^{p(d)}(\boldsymbol{d})$, realised as described in Claim 3.8. That is, we allocate two i.i.d. excess edge-weights $X_{1}^{(e)}, X_{2}^{(e)}$ to each edge $e=(z, w)$, with the meaning that $X_{1}^{(e)}$ belongs to the vertex with smaller index in $[n]$ (say $z$ ), and keep an edge if and only if its excess edge-weights satisfy $X_{1}^{(e)} \leq \xi_{d_{z}}, X_{2}^{(e)} \leq$ $\xi_{d_{w}}$. By Lemma 3.3 and Corollary 3.10, the obtained percolated graph can be coupled to a configuration model $\mathrm{CM}_{n+A}\left(\boldsymbol{d}^{r}, \mathbf{1}(A)\right)$, where again, each half-edge $s$ is kept and stays attached to vertex $x$ if and only if its excess edge-weight $X_{s}$ satisfies $X_{s} \leq \xi_{d_{v(s)}}$, as described in Claim 3.9. Suppose now that we can define a threshold function $\left(\xi_{d}\right)_{d \geq 1}$ that in turn defines $p(d):=\mathbb{P}\left(X \leq \xi_{d}\right)$ as in Claims 3.8 and 3.9, and that $\xi_{d}$ is chosen in a way that $p(d)$ satisfies (3.9). Then, for the empirical degree distribution of $\mathrm{CM}_{n}(\boldsymbol{d}, \mathbf{1}(A))$, Proposition 3.5 applies and the assumptions of Corollary 3.7 are satisfied. Here we note that the vertices $u_{k}$ and $v_{k}$ are not uniformly distributed (as required in Proposition 2.1(a)). However, conditionally on $d_{u_{k}}^{r}=\ell_{1}$, the vertex $u_{k}$ does have a uniform distribution by exchangeability (and the same for $v_{k}$ conditionally on $d_{v_{k}}^{r}=\ell_{2}$ ), so that we are allowed to use Corollary 3.7. 
Corollary 3.7 guarantees the existence of a path $\left(\pi_{i}\right)_{i=1}^{2 b+1}$ with $\pi_{0}=u_{k}$ and $\pi_{2 b+1}=v_{k}$ with $b \leq \log \log n /|\log (\tau-2)|$ within ${\widetilde{\mathrm{CM}_{n}^{(d)}}}^{(d)}$ with probability at least $1-\varepsilon_{3.7}$. So,

$$
W_{n}\left(u_{k}, v_{k}\right) \leq 2 b+1+2 \sum_{i=0}^{b}\left(2 \xi_{\pi_{i}}\right),
$$

where we have noted that the path uses two kept half-edges at each inner vertex $\pi_{i}$ on the path, and the two sides of the path (one from $u_{k}$, one from $v_{k}$ ) constitute to the other factor 2. Further, the event $\mathcal{E}_{n} \in{\widetilde{\mathrm{CM}_{n}^{p}}}^{p(d)}(\boldsymbol{d})$ (see (2.2) and Corollary 3.7) guarantees that $\pi_{i}, i \leq b$ has regular degree at least $d_{\pi_{i}}^{r} \geq\left(k^{1-\delta}\right)^{(\tau-2)^{-i}}$ while $\pi_{i}, i \geq b$ has $d_{\pi_{i}}^{r} \geq\left(k^{1-\delta}\right)^{(\tau-2)^{2 b+1-i}}$ for all $i$. Finally, the at most two connecting vertices within $\Lambda_{\eta}$ have degree at least $n^{\eta}$ and as a result the at most 4 half-edges connected to them on the constructed path have excess half-edge weight at most $\xi_{n^{\eta}}$ (see the proof of Proposition 2.1 below (2.29)). Since $d_{x} \geq d_{x}^{r}$ for all $x$ and we may assume that $\xi_{d}$ is non-increasing in $d$, the excess half-edge weight at $\pi_{i}, i \leq b$ is at most

$$
\xi_{d_{\pi_{i}}} \leq \xi_{\left(k^{1-\delta}\right)^{(\tau-2)}-i}
$$

Combining this with (4.10), with probability at least $1-2 \varepsilon_{(4.10)}-\varepsilon_{3.7}$, and $r=r\left(\varepsilon_{(4.10)}\right)$,

$$
W_{n}(u, v) \leq 2 r+2 \frac{\log \log n}{|\log (\tau-2)|}+1+4 \sum_{i=0}^{\infty} \xi_{\left(k^{1-\delta}\right)^{(\tau-2)}}+4 \xi_{n^{\eta}} .
$$

Note that $4 \xi_{n^{\eta}}<4$ for all sufficiently large $n$. Hence, it remains to show that, when the integrability criterion in (1.6) holds, we can choose, for each $r^{\prime}>0,\left(\xi_{d}\right)_{d \geq 1}$ and $k$ so large that $p(d)=\mathbb{P}\left(X \leq \xi_{d}\right)$ satisfies (3.9) and that

$$
4 \sum_{i=1}^{\infty} \xi_{\left(k^{1-\delta}\right)^{(\tau-2)}-i \wedge(b-i)}<r^{\prime} .
$$

We start by rewriting the integrability criterion in (1.6). By a change of variables $u:=1 / y$, we obtain that (1.6) is equivalent to the convergence of

$$
\int_{0}^{c} F_{X}^{(-1)}(\exp \{-1 / y\}) \frac{1}{y} \mathrm{~d} y
$$

for some $c>0$. For now, let us fix an arbitrary $\gamma \in(\tau-2,1)$ and cut the integral at the powers of $\gamma$. Then, the convergence of (4.15) implies the convergence of the sum

$$
\sum_{n=K}^{\infty} \int_{\gamma^{n+1}}^{\gamma^{n}} F_{X}^{(-1)}(\exp \{-1 / y\}) \frac{1}{y} \mathrm{~d} y
$$

where $K:=\min \left\{n: \gamma^{n} \leq c\right\}$. By the monotonicity of the inverse function $F_{X}^{(-1)}(\cdot)$,

$$
F_{X}^{(-1)}\left(\frac{1}{\mathrm{e}^{1 / \gamma^{n+1}}}\right)(1-\gamma) \leq \int_{\gamma^{n+1}}^{\gamma^{n}} F_{X}^{(-1)}\left(\frac{1}{\mathrm{e}^{1 / y}}\right) \frac{1}{y} \mathrm{~d} y,
$$

hence the convergence of the integral in (1.6) implies that

$$
\sum_{n=K}^{\infty} F_{X}^{(-1)}\left(\frac{1}{\mathrm{e}^{1 / \gamma^{n}}}\right)<\infty
$$


Now we turn to the choice of $\xi_{d}$ and thus $p(d)$. For fixed $r^{\prime}>0$, choose $K\left(r^{\prime}\right)$ so large in (4.18) that the sum on the lhs is at most $r^{\prime} / 4$ :

$$
\sum_{n=K\left(r^{\prime}\right)}^{\infty} F_{X}^{(-1)}\left(\frac{1}{\mathrm{e}^{1 / \gamma^{n}}}\right)=\sum_{n=0}^{\infty} F_{X}^{(-1)}\left(\frac{1}{\mathrm{e}^{1 / \gamma^{n+K\left(r^{\prime}\right)}}}\right)<r^{\prime} / 4 .
$$

The intuitive idea is the following: recall that in the path constructed in the proof of Proposition 2.1, by Lemmas 2.2 and 2.4, for the $i$ th vertex of the constructed path, the degrees are $y_{i} \in\left(\left(k^{1-\delta}\right)^{1 /(\tau-2)^{i}}, k^{1 /(\tau-2)^{i}}\right)$, for some constants $k$ and $\delta \in(0,1)$. Thus, we would like to set $\xi_{d}$ so that the equation

$$
\xi_{\left(k^{1-\delta}\right)^{1 /(\tau-2)^{i}}}=F_{X}^{(-1)}\left(\mathrm{e}^{-1 / \gamma^{i+K\left(r^{\prime}\right)}}\right)
$$

holds, and then (4.14) is satisfied. For this, $d=\left(k^{1-\delta}\right)^{1 /(\tau-2)^{i}}$ implies that $i=$ $\log \left(\log (d) / \log \left(k^{1-\delta}\right)\right) /|\log (\tau-2)|$, which, when used on the right hand side of (4.20), results in the definition

$$
\xi_{d}:=F_{X}^{(-1)}\left(\exp \left\{-\gamma^{-K\left(r^{\prime}\right)}(\log d)^{|\log \gamma| /|\log (\tau-2)|}\left(\log k^{1-\delta}\right)^{-|\log \gamma| /|\log (\tau-2)|}\right\}\right) .
$$

Using that probability distribution functions are right-continous, $F_{X}\left(F_{X}^{(-1)}(x)\right) \geq x$, and hence

$$
\begin{aligned}
p(d) & =F_{X}\left(\xi_{d}\right) \geq \exp \left\{-(\log d)^{|\log \gamma| /|\log (\tau-2)|} \gamma^{-K\left(r^{\prime}\right)}\left(\log k^{1-\delta}\right)^{-\log \gamma /|\log (\tau-2)|}\right\} \\
& =\exp \left\{-C^{\prime}\left(k, r^{\prime}\right)(\log d)^{|\log \gamma| /|\log (\tau-2)|}\right\},
\end{aligned}
$$

Due to the fact that we have chosen $\gamma \in(\tau-2,1)$, we have $|\log \gamma| /|\log (\tau-2)|<1$. Thus, the conditions of Proposition 3.5 are satisfied with this choice of $p(d)$. This, combined with (4.13) finishes the proof of the upper bound. The proof of the lower bound follows from the fact that

$$
W_{n}(u, v) \geq \mathcal{D}_{n}(u, v),
$$

and the latter is lower tight around $2 \log \log n /|\log (\tau-2)|$ by Proposition 1.8.

Acknowledgements This work is supported by the Netherlands Organisation for Scientific Research (NWO) through VICI Grant 639.033.806 (EB and RvdH), VENI Grant 639.031.447 (JK), the Gravitation NETWORKS Grant 024.002.003 (RvdH) and the STAR Cluster (JK).

\section{Compliance to Ethical Standards}

Ethical Standards This work complies with the Ethical Standards of the Journal of Statistical Physics.

Human and Animal Rights In particular, the work did not involve human participants nor animals

Conflict of interest This work does not create any conflict of interest.

Open Access This article is distributed under the terms of the Creative Commons Attribution 4.0 International License (http://creativecommons.org/licenses/by/4.0/), which permits unrestricted use, distribution, and reproduction in any medium, provided you give appropriate credit to the original author(s) and the source, provide a link to the Creative Commons license, and indicate if changes were made. 


\section{References}

1. Adriaans, E., Komjáthy, J.: Weighted distances in scale-free configuration models. J. Stat. Phys. 139, 1-28 (2017)

2. Amini, O., Devroye, L., Griffiths, S., Olver, N.: On explosions in heavy-tailed branching random walks. Ann. Probab. 41(3B), 1864-1899 (2013)

3. Baroni, E., van der Hofstad, R., Komjáthy, J.: Fixed speed competition on the configuration model with infinite variance degrees: unequal speeds. Electron. J. Probab. 20, 1-48 (2015)

4. Baroni, E., van der Hofstad, R., Komjáthy, J.: Nonuniversality of weighted random graphs with infinite variance degree. J. Appl. Probab. 54(1), 146-164 (2017)

5. Bhamidi, S., van der Hofstad, R., Hooghiemstra, G.: First passage percolation on random graphs with finite mean degrees. Ann. Appl. Probab. 20(5), 1907-1965 (2010)

6. Bhamidi, S., van der Hofstad, R., Hooghiemstra, G.: First passage percolation on the Erdős-Rényi random graph. Combin. Probab. Comput. 20(5), 683-707 (2011)

7. Bollobás, B.: Random Graphs. Cambridge University Press, Cambridge (2001)

8. Davies, P.L.: The simple branching process: a note on convergence when the mean is infinite. J. Appl. Probab. 15(3), 466-480 (1978)

9. Federico, L., van der Hofstad, R.: Critical window for connectivity in the configuration model. Combin. Probab. Comput. 26(5), 660-680 (2017)

10. Hammersley, J.M., Welsh, D.J.A.: Bernoulli 1713, Bayes 1763, Laplace 1813: Anniversary Volume. In: Proceedings of an International Research Seminar Statistical Laboratory University of California, Berkeley 1963, pp. 61-110. Springer Berlin (1965)

11. Janson, S.: One, two and three times $\log n / n$ for paths in a complete graph with random weights. Comb. Probab. Comput. 7, 347-361 (1999)

12. Janson, S.: On percolation in random graphs with given vertex degrees. Electron. J. Probab. 14(5), 86-118 (2009)

13. Kaluza, P., Kölzsch, A., Gastner, M.T., Blasius, B.: The complex network of global cargo ship movements. J. R. Soc. Interface 7(48), 1093-1103 (2010)

14. Komjáthy, J.: Explosive Crump-Mode-Jagers branching processes. arXiv:1602.01657 (2016)

15. Sinclair, A.: Class notes for the course "Randomness and Computation". Accessed 12 Sept 2016, Fall 2011

16. van den Esker, H., van der Hofstad, R., Hooghiemstra, G.: Universality for the distance in finite variance random graphs. J. Stat. Phys. 133(1), 169-202 (2008)

17. van der Hofstad, R.: Random Graphs and Complex Networks, vol. 1. Cambridge University Press, Cambridge (2017)

18. van der Hofstad, R.: Stochastic processes on random graphs. 2018+. In: preparation, see http://www.win. tue.nl/ rhofstad/SaintFlour_SPoRG.pdf

19. van der Hofstad, R., Hooghiemstra, G., Znamenski, D.: Distances in random graphs with finite mean and infinite variance degrees. Electron. J. Probab. 12, 703-766 (2007)

20. van der Hofstad, R., Komjáthy, J.: When is a scale-free graph ultra-small? J. Stat. Phys. 169(2), 223-264 (2017) 